\title{
Deformation spaces of trees
}

\author{
Vincent Guirardel and Gilbert Levitt
}

\begin{abstract}
Let $G$ be a finitely generated group. Two simplicial $G$-trees are said to be in the same deformation space if they have the same elliptic subgroups (if $H$ fixes a point in one tree, it also does in the other). Examples include Culler-Vogtmann's outer space and spaces of JSJ decompositions. We discuss what features are common to trees in a given deformation space, how to pass from one tree to all other trees in its deformation space, and the topology of deformation spaces. In particular, we prove that all deformation spaces are contractible complexes.
\end{abstract}

Mathematics Subject Classification (2000). 20E06, 20E08, 20F28, 20 F65.

Keywords. Deformation space, actions on trees, contractible.

\section{Introduction}

Let $G$ be a finitely generated group. A $G$-tree is a simplicial tree with an action of $G$. The notion of a deformation space was introduced by Forester [16]. By definition, two $G$-trees are in the same deformation space $\mathscr{D}$ if they have the same elliptic subgroups: if $H \subset G$ fixes a point in one tree, it also does in the other. Forester showed that two trees in the same $D$ may be connected by a finite sequence of elementary deformations, each associated to a canonical isomorphism $A *_{B} B \simeq A$.

Examples of deformation spaces are Culler-Vogtmann's outer space [12], as well as spaces constructed by McCullough-Miller [26] and the authors [20] to study automorphisms of free products, and the canonical set of splittings of generalized Baumslag-Solitar groups [18], [25].

Deformation spaces are especially relevant to JSJ theory. JSJ decompositions of finitely presented groups have been constructed by Rips-Sela [30], DunwoodySageev [15], Fujiwara-Papasoglu [19]. They are $G$-trees (equivalently, graphs of groups decompositions of $G$ ) with certain properties. Though canonical they are only unique up to certain moves (see the above references, as well as [17], [4], [31]).

We shall explain in [22] (see [21]) that, in general, the canonical object is not a JSJ-tree but a JSJ deformation space, and that a JSJ-tree is just as unique as a tree 
in a general deformation space. We shall also give a general construction of this JSJ space, valid for any finitely presented group and any class of edge groups (not just slender ones).

With this in mind, we study here general properties of deformation spaces, focusing on three main questions:

- Starting with one tree, what moves are needed to generate all trees in its deformation space? Are slide moves sufficient?

- What is common to trees in the same deformation space? In particular, to what extent do trees in the same space have the same vertex and edge stabilizers?

- What is the topology of a deformation space? Is it contractible? Is it finite dimensional, or with a finite dimensional spine?

\section{Contents of the paper}

We fix a finitely generated group $G$ and we consider metric simplicial trees $T$ with an isometric action of $G$, up to equivariant isometry. The action is always assumed to be minimal. In this introduction we also assume irreducibility (it makes several statements simpler).

A subgroup $H \subset G$ is elliptic in $T$ if it fixes a point. By definition, two trees $T, T^{\prime}$ are in the same deformation space $\mathcal{D}$ if they have the same elliptic subgroups. Equivalent characterizations (mainly due to Forester [16]) are the following (see Theorem 3.8): there exist equivariant maps $f: T \rightarrow T^{\prime}$ and $f^{\prime}: T^{\prime} \rightarrow T$; the trees are related by a sequence of elementary deformations; their length functions are biLipschitz equivalent.

We also consider restricted deformation spaces $\mathscr{D}_{\mathscr{A}}$, where we only consider trees with edge stabilizers in a class $\mathcal{A}$ of subgroups of $G$ (such as cyclic subgroups, abelian subgroups, etc.).

Sections 2 and 3 consist mainly of known facts and definitions. In Section 4, we discuss what invariants may be extracted from a given deformation space $\mathfrak{D}$.

For instance, the first Betti number of the quotient graph $\Gamma=T / G$ depends only on $\mathscr{D}$, not on $T$. We denote it by $b_{1}(\mathscr{D})$.

Unfortunately, it is not true that all trees in $\mathscr{D}$ have the same vertex or edge stabilizers. For one thing, one may (almost always) introduce new vertex groups by using the isomorphism $A \simeq A *_{B} B$. This leads us to consider trees which are reduced (in the sense of [16]): if one collapses to a point all edges in a given orbit, one always obtains a tree outside of $\mathscr{D}$.

But reduced trees in $\mathscr{D}$ do not always have the same vertex groups. A basic example is the Baumslag-Solitar group BS $(1,6)=\left\langle a, t \mid t a t^{-1}=a^{6}\right\rangle$ [18]. In the deformation space consisting of trees with all stabilizers cyclic, there exists a reduced tree with vertex stabilizers conjugate to $\langle a\rangle$, and another one exists with stabilizers 
conjugate to $\left\langle a^{2}\right\rangle$. If $G=\operatorname{BS}(2,4)$, there exist a reduced tree with one orbit of vertices and another tree with two orbits.

One way to avoid these problems is to consider a restricted deformation space $\mathscr{D}_{\mathcal{A}}$ and vertex stabilizers not in $\mathcal{A}$. It is then true that all trees in $\mathscr{D}_{\mathcal{A}}$ have the same vertex stabilizers not in $\mathcal{A}$.

In general, the information about vertex stabilizers of trees in $\mathscr{D}$ is captured by a finite set $\mathcal{M}$ which we associate to $\mathcal{D}$.

In nice situations, for instance when no group fixing an edge is properly contained in a conjugate of itself, $\mathcal{M}$ is simply the set of conjugacy classes of vertex stabilizers of reduced trees (see Section 7 for more general statements). In general, an element of $\mathcal{M}$ is a union of conjugacy classes of elliptic subgroups.

If $\mathcal{T} \in \mathscr{D}$ is reduced, we show that the number $s$ of vertices of the quotient graph satisfies $|\mathcal{M}| \leq s \leq 2|\mathcal{M}|+2 b_{1}(\mathscr{D})-2$. This upper bound on $s$ can be viewed as a simple accessibility result, holding within a fixed deformation space (note that $G$ is not assumed to be finitely presented).

Similarly, it is not always true that reduced trees in $\mathscr{D}$ have the same edge groups. But they have the same bi-elliptic groups and the same generalized edge groups, where a group is bi-elliptic if it fixes two distinct points of $T$, is a generalized edge group if it is bi-elliptic and furthermore it contains some edge stabilizer.

In JSJ theory, one encounters quadratically hanging subgroups. The important information about such a subgroup is not its isomorphism type (it is often a free group), but the way it is attached to the rest of the group (the topological picture being a compact surface attached along its boundary components). It is therefore desirable to attach a peripheral structure to vertex stabilizers of trees in $\mathscr{D}$.

We explain how to define such a structure, given $\mathscr{D}$ and a maximal elliptic subgroup $G_{0}$. All trees in $\mathscr{D}$ have a vertex $v$ with stabilizer $G_{0}$, and the peripheral structure contains information about incident edge groups. In nice situations, it is simply the set of conjugacy classes of stabilizers of edges incident to $v$.

In Section 5, we discuss topologies on a deformation space $\mathcal{D}$. There are two natural topologies on $\mathscr{D}$. The first topology is the equivariant Gromov-Hausdorff topology (or simply Gromov topology), also called the axes topology because it may be described in terms of length functions. The second topology is the weak topology, associated to the natural structure of a cell complex on $\mathcal{D}$.

As pointed out in [26], these topologies may be different. We show, however, that they always agree on any subset of $\mathscr{D}$ which is contained in a finite union of cells, and also show that they agree on the whole of $\mathscr{D}$ when $\mathscr{D}$ consists of locally finite trees with finitely generated stabilizers. (As mentioned above, we assume in this introduction that trees are irreducible. When $\mathscr{D}$ consists of $G$-trees with a $G$-fixed end, we have to show the non-obvious fact that $\mathscr{D}$ is Hausdorff in the Gromov topology).

In Section 6, we discuss contractibility. Skora [35] introduced the idea of deforming morphisms to prove that Culler-Vogtmann space and its closure are contractible. 
Using his technique, we show that a deformation space $\mathscr{D}$ is always contractible in the weak topology. When $\mathscr{D}$ contains a tree with finitely generated vertex groups, we show that it is contractible in the Gromov topology (this was proved independently by Clay [7]) and the closure of $\mathscr{D}$ is contractible.

Here is a sketch of the argument for proving contractibility. It is relatively easy to construct a map $F: \mathscr{D} \times[0,1] \rightarrow \mathscr{D}$ contracting $\mathscr{D}$ to a point (this requires choosing a basepoint in trees of $\mathscr{D}$ in a continuous way; we give a direct geometric construction, different from Skora's minimization argument). The problem is to show that $F$ is continuous. We consider its restriction to $\delta \times[0,1]$, where $\delta$ is a closed cell. It is continuous in the Gromov topology. We show that the image of this restriction is a subset of $\mathscr{D}$ meeting only finitely many cells (Finiteness Lemma 6.4). Since the two topologies agree on such a subset, $F$ is continuous in the weak topology on $8 \times[0,1]$, hence on the whole of $\mathscr{D} \times[0,1]$ by definition of the weak topology. As in [7], proving continuity in the Gromov topology requires a finiteness hypothesis.

We also use Skora's technique to give a direct proof of Forester's deformation theorem [16].

In Section 7, we study a "nice" class of deformation spaces, the non-ascending ones. Without giving the definition here, let us mention two special cases. First, spaces containing a tree which is weakly acylindrical in the following sense: if $g \in G$ is non-trivial, its fixed point set contains no infinite ray. Also, spaces consisting of trees such that no edge stabilizer properly contains a conjugate of itself. This applies in particular to splittings over finite subgroups and to cyclic splittings of groups containing no solvable Baumslag-Solitar group BS $(1, n)$ with $n \geq 2$.

Generalizing a result of Forester, we show that any two reduced trees in a nonascending deformation space are related by slide moves. In particular, they have exactly the same vertex and edge stabilizers. We also show that a non-ascending deformation space has a natural deformation retraction onto a finite-dimensional subcomplex.

In Section 8, we consider a finitely generated subgroup $F$ of $\operatorname{Out}(G)$ leaving a deformation space $\mathscr{D}$ invariant (see [5], [8], [23], [36] for related results). We show that the fixed point set of $F$ in $D$ is empty or contractible, using the fact that this fixed point set is a deformation space of $\widehat{G}$-trees for some extension $\widehat{G}$ of $G$. If $F$ is a solvable finite group and $\mathscr{D}$ is non-ascending, we show that $F$ does have a fixed point in $\mathscr{D}$. We conclude the paper by a few facts about automorphisms leaving invariant a deformation space consisting of locally finite trees.

\section{Trees}

Let $G$ be a finitely generated group. Unless otherwise indicated (in Sections 5 and 6), all trees will be simplicial $G$-trees, i.e., we consider a simplicial tree $T$ with an action 
of $G$ by simplicial automorphisms, without inversions. See [6], [11], [34] for basic facts about trees.

We always assume that $T$ is minimal: there is no proper $G$-invariant subtree. This implies that there are only finitely many orbits of edges, and no terminal vertices.

We usually assume that there is no redundant vertex (every vertex of $T$ has degree $\geq 3$ ), though we sometimes need to subdivide trees (in the proof of Lemma 6.5, for instance).

Two distinct points $a, b$ of $T$ bound a unique segment $[a, b]$. A finite subtree is the convex hull of a finite set of points. An end of $T$ is an equivalence class of infinite rays, two rays being equivalent if their intersection is a subray.

A $G$-tree $T$ may be considered as a simplicial (or combinatorial) object, or as a metric space with an isometric action of $G$. The simplest way to define a metric is to assign length 1 to every edge. More generally, one may assign any positive length to each orbit of edges (recall that there are finitely many orbits).

Different length assignments lead to metric trees with the same underlying simplicial tree (in Section 5, we shall say that they belong to the same open cone). They are equivariantly bi-Lipschitz homeomorphic. It will sometimes be important to distinguish between a metric tree and the underlying simplicial (non-metric) tree.

Let $T$ be a metric $G$-tree. The length function $\ell_{T}: G \rightarrow \mathbb{R}$ is defined by $\ell_{T}(g)=$ $\min _{x \in T} d(x, g x)$. When no confusion is possible, we simply write $\ell$. An element $g \in G$ is elliptic if it has a fixed point (equivalently, if $\ell(g)=0$ ), hyperbolic otherwise. A hyperbolic element has a translation axis $A_{g}$, on which it acts as translation by $\ell(g)$ and $d(x, g x)=\ell(g)+2 d\left(x, A_{g}\right)$ for any $x \in T$. This formula also holds if $g$ is elliptic, with $A_{g}$ understood as the fixed point set of $g$.

A subgroup $H \subset G$ is elliptic if it fixes a point. A finitely generated subgroup is elliptic if and only if all its elements are elliptic [33]. But an infinitely generated subgroup consisting of elliptic elements may fail to be elliptic; it then fixes a unique end of $T$ (see for instance [6], Theorem 3.2.6).

We distinguish five types of minimal $G$-trees (see [11] or [6], p. 134):

- Trivial: $T$ is a point.

- Dihedral: $T$ is a line, but $G$ does not preserve orientation. The action factors through an action of the infinite dihedral group $D_{\infty} \simeq \mathbb{Z} / 2 \mathbb{Z} * \mathbb{Z} / 2 \mathbb{Z}$.

- Linear abelian: $T$ is a line, and $G$ acts by translations. The action factors through an action of $\mathbb{Z}$.

- Genuine abelian: $G$ fixes an end of $T$, and $T$ is not a line. The quotient graph $\Gamma=T / G$ is homeomorphic to a circle. When there is only one orbit of edges, $T$ is the Bass-Serre tree of a strictly ascending HNN-extension $G=\langle A, t|$ tat $\left.^{-1}=\varphi(a)\right\rangle$, where $\varphi: A \rightarrow A$ is injective but not onto.

- Irreducible: there exist two hyperbolic elements with disjoint axes. In this case, $G$ contains a free group of rank 2 acting freely. 
Knowing the partition of $G$ into elliptic and hyperbolic elements (i.e. the zeroset of the length function) is enough to distinguish trivial, dihedral, abelian, irreducible trees [11] (but the two abelian types are not distinguished). In particular, $T$ is irreducible if and only if there exist hyperbolic elements $g, h$ whose commutator $[g, h]=g h g^{-1} h^{-1}$ is hyperbolic.

A tree is abelian if and only if its length function is the absolute value of a homomorphism from $G$ to $\mathbb{R}$ with cyclic image. Every element of the commutator subgroup $[G, G]$ is elliptic, but the subgroup $[G, G]$ is elliptic if and only if the tree is linear abelian. In particular, there is no genuine abelian $G$-tree if $[G, G]$ is finitely generated.

Trees are considered equal if they are equivariantly isometric. Two minimal nonabelian $G$-trees with the same length function are equivariantly isometric (see [11]).

Maps between $G$-trees will always be $G$-equivariant. As trees are minimal, maps are always onto. A map $f: T \rightarrow T^{\prime}$ between simplicial trees is simplicial if each edge is mapped bijectively onto an edge (in particular, no edge is collapsed). It is a morphism if each edge of $T$ can be written as a finite union of subsegments, each of which is mapped bijectively onto a segment in $T^{\prime}$. Equivalently, $f$ is a morphism if and only if one may subdivide $T$ and $T^{\prime}$ so that $f$ becomes simplicial. When $T$ and $T^{\prime}$ are metric trees, simplicial maps and morphisms are required to map the subsegments isometrically.

\section{Generalities on deformation spaces}

We fix a finitely generated group $G$. All trees considered in this section will be minimal metric simplicial $G$-trees, up to equivariant isometry. We always assume that $G$ acts without inversions. In this section and in the next one, only the simplicial structure will really matter. In later sections, however, allowing edge lengths to vary will be crucial.

Definition 3.1 (domination). We say that $T$ dominates $T^{\prime}$ if there is an equivariant (but otherwise arbitrary) map $f$ from $T$ to $T^{\prime}$.

Equivalently, $T$ dominates $T^{\prime}$ if and only if every elliptic subgroup of $T$ is elliptic in $T^{\prime}$. The "only if" direction is clear and the converse is proved by first defining $f$ equivariantly on vertices, and then extending linearly to edges. Domination is a pre-ordering. The corresponding equivalence classes are deformation spaces:

Definition 3.2 (deformation space). The deformation space $\mathscr{D}$ containing $T$ is the set of metric trees $T^{\prime}$ such that $T$ and $T^{\prime}$ dominate each other, up to equivariant isometry. Trees are in the same $\mathscr{D}$ if and only if they have the same elliptic subgroups. Identify- 
ing two trees when they differ only by rescaling the metric leads to the projectivized deformation space P D.

For now $\mathcal{D}$ and $P \mathscr{D}$ are just sets, with no extra structure, but in Section 5 we shall view them as topological spaces and complexes.

Definition 3.3 (collapse, collapsible edge). Let $e$ be an edge of $T$. Collapsing every edge in the orbit of $e$ to a point produces a new tree $T^{\prime}$. We say that $T^{\prime}$ is obtained by collapsing $T$ (or $e$ ), and $T$ is obtained by expanding $T^{\prime}$. Collapsing is defined for simplicial trees. In the context of metric trees, it may be viewed as assigning length 0 to edges in the orbit of $e$.

The tree $T^{\prime}$ is dominated by $T$. Even if $T^{\prime}$ is not trivial, it is not necessarily in the same $\mathscr{D}$ as $T$ (this may happen if $T$ is irreducible). We say that $e$ is collapsible if $T$ and $T^{\prime}$ are in the same deformation space.

The stabilizer of a point $v$, or an edge $e=v w$, will be denoted by $G_{v}$ or $G_{e}$. We note that an edge $e=v w$ is collapsible if and only if $v$ and $w$ are in distinct orbits and at least one of the inclusions from $G_{e}$ to $G_{v}$ or from $G_{e}$ to $G_{w}$ is onto [16].

Indeed, if $v$ and $w$ are in distinct orbits and the inclusion from $G_{e}$ to $G_{v}$ is onto, then collapsing $e$ does not change the set of elliptic subgroups: the stabilizer of $w$ does not change ( $\operatorname{since} G_{v} *_{G_{v}} G_{w}=G_{w}$ ), and although the vertex $v$ "disappears" its stabilizer remains elliptic (it is contained in $G_{w}$ ). On the other hand, collapsing $e$ with $G_{e} \varsubsetneqq G_{v}$ and $G_{e} \varsubsetneqq G_{w}$ would create a new elliptic subgroup, namely $G_{v} *_{G_{e}} G_{w}$. If $v$ and $w$ are in the same orbit, say $g v=w$, then $g$ is hyperbolic in $T$ (because there is no inversion) and collapsing $e$ makes $g$ elliptic.

Definition 3.4 (elementary collapse, elementary deformation). When $e$ is collapsible, passing from $T$ to $T^{\prime}$ is called an elementary collapse. The reverse of an elementary collapse is an elementary expansion. A finite sequence of elementary collapses and expansions is called an elementary deformation [16]. An elementary deformation between metric trees is an elementary deformation between the underlying simplicial trees.

Definition 3.5 (reduced). A tree $T$ is reduced (in the sense of Forester [16]) if no elementary collapse is possible (collapsing any edge yields a tree in a different deformation space). Equivalently: if an edge $e=v w$ satisfies $G_{e}=G_{v}$, then $v$ and $w$ are in the same $G$-orbit.

Being reduced is stronger than being $B F$-reduced (in the sense of Bestvina-Feighn [3]), where $G_{e} \rightarrow G_{v}$ being onto only implies that the image of $v$ in the quotient graph $\Gamma=T / G$ has valence at least 3 .

Given any tree $T$, one may perform a (usually non-unique) sequence of elementary collapses so as to obtain a reduced tree $T_{r}$. We say that $T_{r}$ is a reduction of $T$. 
Remark 3.6. If the reduced trees $T, T^{\prime}$ are related by an elementary deformation, there exists a sequence of reduced trees $T=T_{1}, \ldots, T_{k}=T^{\prime}$ such that $T_{i}$ and $T_{i+1}$ are different reductions of a tree $S_{i}$ (to see this, call $S_{i}$ the intermediate trees appearing in the deformation between $T$ and $T^{\prime}$, and choose a reduction $T_{i}$ for each $S_{i}$ ).

Remark 3.7. Suppose that $T, T^{\prime}$ have the same elliptic subgroups, and $T$ is reduced. If $f: T \rightarrow T^{\prime}$ is equivariant, and $e=v w$ is an edge of $T$, then $f(v) \neq f(w)$. Otherwise, one could modify $f$ to be constant on $e$, so $f$ would factor through the tree $T^{\prime \prime}$ obtained by collapsing $e$. But since $T$ is reduced, $T^{\prime \prime}$ does not dominate $T$. Since $T^{\prime \prime}$ dominates $T^{\prime}$ which dominates $T$, this is a contradiction.

By definition, trees related by an elementary deformation are in the same deformation space. Forester proved that the converse is true, hence the terminology. More explicitly:

Theorem 3.8. Given two $G$-trees $T$ and $T^{\prime}$, the following are equivalent:

(1) $T$ and $T^{\prime}$ are in the same deformation space;

(2) there exist equivariant maps $f: T \rightarrow T^{\prime}$ and $f^{\prime}: T^{\prime} \rightarrow T$;

(3) $T$ and $T^{\prime}$ have the same elliptic subgroups;

(4) $T$ and $T^{\prime}$ are related by an elementary deformation;

(5) there exists an equivariant quasi-isometry from $T$ to $T^{\prime}$;

(6) there exists an equivariant map $f: T \rightarrow T^{\prime}$ having bounded preimages.

If $T$ and $T^{\prime}$ are not abelian one may add:

(7) the length functions $\ell_{T}, \ell_{T^{\prime}}$ of $T$ and $T^{\prime}$ are bi-Lipschitz equivalent (there exists $C>0$ such that $\left.\frac{1}{C} \ell_{T} \leq \ell_{T^{\prime}} \leq C \ell_{T}\right)$.

All conditions are obviously satisfied when $T, T^{\prime}$ are metric trees with the same underlying simplicial tree, so this is really a statement about simplicial trees.

Proof. The equivalence between (1) through (6) is in [16]. We sketch some of the arguments. We have seen (1) $\Leftrightarrow(2) \Leftrightarrow$ (3). The implication (3) $\Rightarrow$ (4) is one of the main results of [16]; we shall provide a new proof in Section 6 (see Theorem 6.6). $(4) \Rightarrow(5)$ because an elementary collapse is a quasi-isometry: if $e$ is a collapsible edge, then any connected component of G.e has diameter at most 2. Obviously $(5) \Rightarrow$ (6). We show (6) $\Rightarrow$ (3). If $H \subset G$ fixes $x \in T$, it fixes $f(x) \in T^{\prime}$. Conversely, if $H$ fixes $y \in T^{\prime}$, it leaves invariant the bounded set $f^{-1}(y) \subset T$ and so fixes a point in $T$. Thus $(6) \Rightarrow$ (3).

We now show (5) $\Leftrightarrow$ (7). Given any $x \in T$ and $g \in G$, one has $\ell_{T}(g)=$ $\lim _{n \rightarrow \infty} \frac{d\left(x, g^{n} x\right)}{n}$, so (5) always implies (7). Conversely, assume (7). Then $T$ and $T^{\prime}$ 
have the same elliptic elements. We must show that they have the same elliptic subgroups, provided that they are not abelian. We suppose that a subgroup $H$ fixes $y \in T^{\prime}$ but is not elliptic in $T$, and we obtain a contradiction.

The group $H$ fixes a unique end of $T$. Since $T$ is not abelian, it is irreducible and we can find a hyperbolic $g \in G$ whose axis $A_{g}$ does not contain that end. The set $\ell_{T}(g H)$ is then unbounded, as there exist elements of $H$ whose fixed point set is arbitrarily far from $A_{g}$ (see [28], Proposition 1.7). By (7), the set $\ell_{T^{\prime}}(g H)$ also is unbounded. This is a contradiction, as it is bounded by the distance from $y$ to $g y$ in $T^{\prime}$.

Remark 3.9. (3) $\Rightarrow(5)$ and (3) $\Rightarrow$ (6) may obviously be strengthened as follows: if $T, T^{\prime}$ have the same elliptic subgroups, then any equivariant map $f: T \rightarrow T^{\prime}$ which is piecewise linear on each edge is a quasi-isometry and has bounded preimages. We give a direct argument to show that $f$ has bounded preimages if (3) holds (this will be useful in the proof of Theorem 6.6). One can subdivide $T$ and $T^{\prime}$ so that $f$ maps each edge to a vertex or an edge. We only need to prove that the preimage of a vertex is bounded, and since $T, T^{\prime}$ have finitely many orbits of vertices, it suffices to show that sets of the form G.v $\cap f^{-1}(f(v))$ are bounded. This intersection is equal to $H . v \cap f^{-1}(f(v))$, where $H$ is the stabilizer of $f(v)$. By (3), $H$ is elliptic in $T$, so $H . v$ is bounded.

Proposition 3.10. The following statements hold:

(1) All trees in a given deformation space have the same type (trivial, dihedral, trivial abelian, genuine abelian, irreducible).

(2) All trees in a given abelian deformation space have the same length function up to scaling.

(3) If $T$ is trivial, linear abelian, or dihedral, its projectivized deformation space consists of only one point.

A deformation space $\mathscr{D}$ will be called genuine abelian, or irreducible, if the trees contained in $\mathscr{D}$ are genuine abelian, or irreducible. These are the only interesting types.

Remark 3.11. More generally, a non-elementary collapse does not change the type, as long as the collapsed tree is not trivial.

Proof. The first assertion follows from remarks made in Section 2.

The length function of an abelian tree is the absolute value of a homomorphism $\varphi: G \rightarrow \mathbb{R}$ with cyclic image. The deformation space determines the kernel of $\varphi$, hence $\varphi$ up to scaling. This shows (2).

(3) is trivial in the trivial case. There is only one linear abelian tree with length function $|\varphi|$, so (3) holds in the linear abelian case. Now suppose that $T$ is dihedral, 
and $T^{\prime}$ is in the same deformation space. The actions of $G$ on $T$ and $T^{\prime}$ have the same kernel (elements acting as the identity), equal to the set of elliptic elements whose product with any elliptic element is elliptic. One then observes that the infinite dihedral group only has one non-trivial action, up to scaling.

A deformation space $\mathscr{D}$ is determined by the family $\mathscr{E}$ consisting of all elliptic subgroups. Often one also wants to restrict edge stabilizers. Let $\mathcal{A}$ be a family of subgroups of $G$, stable under conjugating and passing to a subgroup. For instance, $\mathcal{A}$ may consist of all subgroups which are finite, cyclic, abelian, slender, small, etc.

Definition 3.12 (restricted deformation space $\mathscr{D}_{\mathcal{A}}$ ). We define $\mathscr{D}_{\mathcal{A}} \subset D$ as the set of trees $T \in \mathscr{D}$ whose edge stabilizers belong to $\mathcal{A}$. We call $\mathscr{D}_{\mathcal{A}} \subset \mathscr{D}$ a restricted deformation space, or simply a deformation space. We shall see later that any $\mathscr{D}$ contains a smallest nonempty $\mathscr{D}_{\mathscr{A}}$, called the reduced deformation space $\mathfrak{D}_{r}$.

Examples. - If $G$ is a free group $F_{n}$, Culler-Vogtmann's outer space is the projectivized deformation space obtained when $\mathscr{E}$ consists only of the trivial group.

- Suppose that $G=G_{1} * \cdots * G_{p} * \mathbb{Z} * \cdots * \mathbb{Z}$, where each $G_{i}$ is non-trivial, non-isomorphic to $\mathbb{Z}$, and freely indecomposable. Let $\mathcal{E}$ consist of subgroups of conjugates of the factors, and $\mathcal{A}$ consist only of the trivial group. The projectivized space $P \mathscr{D}_{\mathcal{A}}$ is the space constructed by McCullough-Miller [26]. If $\mathscr{E}$ contains only subgroups of conjugates of the factors $G_{i}$, then $P D_{\mathcal{A}}$ is the space considered in [20].

- Let $G$ be a generalized Baumslag-Solitar group (GBS group), i.e., $G$ acts on a tree with all edge and vertex stabilizers isomorphic to $\mathbb{Z}$. If $G$ is not $\mathbb{Z}, \mathbb{Z}^{2}$, or the Klein bottle group, all such $G$-trees belong to the same deformation space [16]. We shall call it the canonical deformation space of $G$. It is genuine abelian if $G$ is a solvable Baumslag-Solitar group $\mathrm{BS}(1, n)$ with $n \neq \pm 1$, irreducible if not. If $G=\mathbb{Z}^{2}$ there are infinitely many deformation spaces, all of them linear abelian. If $G$ is the Klein bottle group, there are two spaces; one is linear abelian, the other one is dihedral.

- Let $G$ be the fundamental group of a closed orientable surface $\Sigma$. Any $G$-tree with cyclic edge stabilizers is dual to a family of disjoint essential simple closed curves (see [27], Theorem III.2.6). After projectivization, the space of metric $G$-trees with cyclic edge stabilizers may be identified with the curve complex of $\Sigma$. Two such trees are in the same deformation space if and only if they belong to the same open simplex.

- Suppose that $G$ has a non-trivial center $Z$, and $\mathscr{D}$ is genuine abelian or irreducible. Standard arguments show that $Z$ acts as the identity on any $T \in \mathscr{D}$ (an element $z \in Z$ cannot be hyperbolic, as its axis would be an invariant line; so $z$ is elliptic, and its fixed point set is an invariant subtree, equal to $T$ by minimality). We may therefore view $\mathscr{D}$ as a deformation space over $G / Z$. 


\section{Invariants of deformation spaces}

Trees in a given deformation space $\mathscr{D}$ may be fairly different. Still, certain features depend only on $\mathscr{D}$.

Betti number. The quotient of $G$ by the subgroup generated by all elliptic elements is a free group, isomorphic to the (topological) fundamental group of the quotient graph $\Gamma=T / G$ for any $T \in \mathscr{D}$. The rank of this group will be called the Betti number $b_{1}(D)$ of $\mathscr{D}$.

Acylindricity. A $G$-tree $T$ is called acylindrical [32] if there exists $k$ such that, for any non-trivial $g \in G$, the fixed point set of $g$ has diameter less than $k$. As all trees in $\mathscr{D}$ are equivariantly quasi-isometric to each other, acylindricity depends only on $\mathscr{D}$ (but $k$ depends on $T$ ).

Similar considerations apply to the following weak form of acylindricity: if $g$ is non-trivial, its fixed point set contains no infinite ray.

Local finiteness. It is easy to see that an elementary collapse, or an elementary expansion, preserves the property that $T$ is locally finite (recall that we only consider minimal trees). Local finiteness of trees is therefore a property of the deformation space. For instance Culler-Vogtmann space, the space constructed by McCulloughMiller when $G$ is a free product of finite groups, the canonical deformation space of a GBS group have this property.

Vertex groups. Though all trees in a deformation space $\mathscr{D}$ have the same elliptic subgroups, they do not necessarily have the same vertex and edge stabilizers, even if they are reduced.

Examples. - In the canonical deformation space of BS $(1,6)=\left\langle a, t \mid t a t^{-1}=a^{6}\right\rangle$, there exists a tree $T_{1}$ whose vertex stabilizers are conjugates of $\langle a\rangle$, and another tree $T_{2}$ whose vertex stabilizers are conjugates of $\left\langle a^{2}\right\rangle$ (see Example 4.3 in [18]). Note that $\langle a\rangle$ is conjugate to the subgroup $\left\langle a^{6}\right\rangle \subset\left\langle a^{2}\right\rangle$.

- Now consider the Bass-Serre tree of the HNN-extension BS $(2,4)=$ $\left\langle a, t \mid t a^{2} t^{-1}=a^{4}\right\rangle$. There is one orbit of vertices. Vertex stabilizers are conjugates of $\langle a\rangle$. On the other hand, the tree associated to the presentation $\operatorname{BS}(2,4)=$ $\left\langle a, b, t \mid t b t^{-1}=b^{2}, a^{2}=b^{2}\right\rangle$, though reduced, has two orbits of vertices, with vertex stabilizers conjugates of $\langle b\rangle$ and $\langle a\rangle$ (see [25]).

These examples are possible because there exist edge stabilizers which properly contain conjugates of themselves. In Section 7, we shall study non-ascending deformation spaces, where such examples cannot occur. In a non-ascending space $\mathscr{D}$, every elliptic subgroup is contained in a maximal one. All reduced trees $T \in \mathscr{D}$ have 
the same vertex and edge stabilizers, and there is a natural bijection between the set of $G$-orbits of vertices and the set $\mathcal{M}$ of conjugacy classes of maximal elliptic subgroups (but there may exist distinct vertices of $T$ with the same stabilizer).

In a general deformation space, we have to define a set $\mathcal{M}$ in a more complicated way.

Definition 4.1 (vertical set $\mathcal{M}$ ). We associate a set $\mathcal{M}$ to $\mathscr{D}$ as follows (compare the definition of vertical subgroups in [18]). Given elliptic subgroups $H$ and $K$, define $H \leq K$ if $H$ is contained in a conjugate of $K$, and $H \sim K$ if $H \leq K$ and $K \leq H$. The set $\mathcal{C}$ of equivalence classes is partially ordered, and we define $\mathcal{M}$ as the set of maximal elements of $\ell$.

If $H$ is a maximal elliptic subgroup, its equivalence class equals its conjugacy class and is an element of $\mathcal{M}$; in general, an element of $\mathcal{M}$ is a union of conjugacy classes of elliptic subgroups. If $\mathcal{D}$ contains $T$ such that no edge-to-vertex inclusion $G_{e} \rightarrow G_{v}$ is onto, $\mathcal{M}$ is simply the set of conjugacy classes of maximal elliptic subgroups; it may be identified with the set of $G$-orbits of vertices.

Example. In the example of $\operatorname{BS}(1,6)$ given above, the groups $\langle a\rangle$ and $\left\langle a^{2}\right\rangle$ are equivalent. Their class is the unique element of $\mathcal{M}$. There is no maximal elliptic subgroup (in this example $\mathcal{D}$ is abelian, but this may also occur in irreducible deformation spaces). In the $\operatorname{BS}(2,4)$ example, the set $\mathcal{M}$ has one element, the conjugacy class of $\langle a\rangle$ (a maximal elliptic subgroup). The vertex stabilizer $\langle b\rangle$ is not maximal elliptic.

Given a tree $T$, we denote by $s$ the number of vertices of the quotient graph $\Gamma=T / G$. It is the number of $G$-orbits of vertices of $T$.

Proposition 4.2. Let $\mathcal{D}$ be a deformation space.

(1) The set $\mathcal{M}$ is finite. If $T \in \mathcal{D}$, the number $s$ of vertices of $\Gamma=T / G$ satisfies $s \geq|\mathcal{M}|$.

(2) If $T \in \mathscr{D}$ is reduced, or only BF-reduced, then $s \leq 2|\mathcal{M}|+2 b_{1}(\mathcal{D})-2$.

Proof. Fix $T \in \mathscr{D}$. There is a natural map $\varphi$ from the set of $G$-orbits of vertices of $T$ to $C$ : associate to the orbit of $v \in T$ the equivalence class of $G_{v}$. Since any elliptic subgroup is contained in some $G_{v}$, the image of $\varphi$ contains $\mathcal{M}$. This shows the first assertion. Also note that any elliptic subgroup is contained in an elliptic subgroup whose class is in $\mathcal{M}$.

The graph $\Gamma$ has first Betti number $b_{1}(\mathcal{D})$. To prove the second assertion, it suffices to show that $|\mathcal{M}|$ is an upper bound for the number of vertices of valence 1 or 2. Suppose that $v \in T$ projects onto such a vertex $\bar{v}$. Since $T$ is minimal and BF-reduced, the group $G_{v}$ is a maximal elliptic subgroup, as no inclusion $G_{e} \rightarrow G_{v}$ is onto. Thus $\varphi(G v) \in \mathcal{M}$. Furthermore, $\varphi(G v) \neq \varphi(G w)$ if $\bar{v}, \bar{w}$ are distinct vertices of valence 1 or 2 . This shows the required bound. 
Assertion (2) of the proposition says that accessibility always holds within a given deformation space (the only assumption being $G$ finitely generated). The accessibility theorem of [3] is equivalent to saying that, given a finitely presented group $G$, there exists a uniform bound $|\mathcal{M}| \leq C$, valid for all deformation spaces containing a tree with small edge stabilizers.

The trees such that $s=|\mathcal{M}|$ are precisely the fully reduced trees defined in [18]. Forester proved that the canonical deformation space of a generalized BaumslagSolitar group always contains a fully reduced tree [18]. He has an example showing that this is not true in general. In any case, even when there is a fully reduced tree, it may not be the most natural element of $\mathcal{D}$.

Example. Let $G=\left\langle a_{1}, b_{1}, a_{2}, b_{2}, a_{3}, b_{3} \mid\left[a_{1}, b_{1}\right]=\left[a_{2}, b_{2}\right]=\left[a_{3}, b_{3}\right]\right\rangle$ be the fundamental group of the space obtained by gluing three once-punctured tori together along their boundary. Consider the associated deformation space, where a subgroup is elliptic if and only if it is contained in a conjugate of $G_{i}=\left\langle a_{i}, b_{i}\right\rangle$. The set $\mathcal{M}$ has 3 elements. There are trees whose quotient graph $\Gamma$ has 3 vertices and 2 edges (for instance the tree corresponding to $G=G_{1} *_{Z} G_{2} *_{Z} G_{3}$, with $Z=\left\langle\left[a_{i}, b_{i}\right]\right\rangle$ ), but none of them is invariant under the automorphism of order 3 mapping $a_{i}, b_{i}$ to $a_{i+1}, b_{i+1} \bmod 3$. To get an invariant (but not reduced) JSJ tree, one has to create a "central" vertex of $\Gamma$ with group $Z$ (see [4]).

As mentioned above, it is not always true that elements of $\mathcal{M}$ are conjugacy classes of maximal elliptic subgroups and correspond bijectively with the vertices of $\Gamma$ for $T$ reduced. Things become nicer in a non-ascending space (see Section 7), or if we work in a restricted deformation space $\mathscr{D}_{\mathcal{A}}$ (see Definition 3.12) and consider only vertex stabilizers not in $\mathcal{A}$.

Proposition 4.3. For any $T \in \mathscr{D}_{\mathcal{A}}$, the assignment $v \mapsto G_{v}$ induces a bijection from the set of vertices $v$ of $T$ with $G_{v} \notin \mathcal{A}$, to the set of maximal elliptic subgroups not in $A$.

If we call big a group which is not in $\mathcal{A}$, we get:

Corollary 4.4. All trees in $\mathscr{D}_{\mathcal{A}}$ have the same “big” vertex stabilizers.

Edge groups. We associate three families of subgroups of $G$ to a $G$-tree $T$. Each family is contained in the next.

- Edge groups: a stabilizer $G_{e}$, with $e$ an edge.

- Generalized edge groups: a group $H$ such that $G_{e} \subset H \subset G_{e^{\prime}}$, with $e, e^{\prime}$ edges of $T$.

- Bi-elliptic groups: a group $H$ contained in some $G_{e}$ (equivalently, a group fixing two distinct points of $T$ ). 
Example. Consider the trees $T_{1}, T_{2}$ in the canonical deformation space of $\operatorname{BS}(1,6)$, as in the examples on page 145. Fix $i \in \mathbb{Z}$. The group $H=\left\langle a^{i}\right\rangle$ is always bi-elliptic (in both $T_{1}$ and $T_{2}$ ). It is a generalized edge group if and only if $i$ divides a power of 6 . It is an edge group of $T_{1}$ if and only if $i$ is a power of 6 , an edge group of $T_{2}$ if and only if $i$ is twice a power of 6 . This example illustrates the general fact that bi-elliptic groups and generalized edge groups are more canonical than edge groups (see Proposition 4.6).

Proposition 4.5. Let $T$ be reduced. A subgroup $H \subset G$ is bi-elliptic in $T$ if and only if it is contained in a subgroup $K$ having one of the following forms:

(1) $K=A \cap B$, where $A$ and $B$ are elliptic, but $\langle A, B\rangle$ is not elliptic.

(2) $K$ is elliptic and there exists a hyperbolic $g \in G$ such that $K \subset g \mathrm{Kg}^{-1}$.

In particular, all reduced trees in a given deformation space have the same bielliptic subgroups.

Proof. We first show that any $K$ as indicated is bi-elliptic. If $K$ is as in (1), the groups $A$ and $B$ fix distinct points $a, b$, and $K$ fixes the segment $[a, b]$. If $K$ is as in (2), it fixes a point $x$ and also the point $g x \neq x$.

Conversely, suppose $H$ fixes an edge $e=a b$. If $G_{e}$ is properly contained in both $G_{a}$ and $G_{b}$, then (1) holds with $K=G_{e}=G_{a} \cap G_{b}$ (note that $\left\langle G_{a}, G_{b}\right\rangle$ is not elliptic). If, say, $G_{e}=G_{a}$, there exists a hyperbolic $g$ such that $b=g a$ (because $T$ is reduced) and (2) holds with $K=G_{a}$.

Proposition 4.6. Given simplicial (non-metric) trees $T, T^{\prime}$ in the same deformation space, the following conditions are equivalent:

(1) There exist morphisms $f: T \rightarrow T^{\prime}$ and $f^{\prime}: T^{\prime} \rightarrow T$.

(2) $T$ and $T^{\prime}$ have the same generalized edge groups.

(3) $T$ and $T^{\prime}$ have the same bi-elliptic groups.

These conditions are satisfied if $T$ and $T^{\prime}$ are reduced.

See Section 2 for the definition of a morphism.

Proof. Clearly (2) $\Rightarrow$ (3), as bi-elliptic groups are just subgroups of generalized edge groups. It is also clear that $(1) \Rightarrow(3)$, since a morphism cannot collapse an edge. Let us show the stronger statement $(1) \Rightarrow(2)$. We have to prove that every edge stabilizer $G_{e}$ of $T$ contains an edge stabilizer of $T^{\prime}$. Since $f^{\prime}$ is onto, some edge $e^{\prime}$ of $T^{\prime}$ contains a non-degenerate segment which is mapped injectively into $e$ by $f^{\prime}$. The stabilizer of $e^{\prime}$ is contained in $G_{e}$.

We now show (3) $\Rightarrow(1)$. Given $T$ and $T^{\prime}$ in $\mathscr{D}$, there is an equivariant map $f: T \rightarrow T^{\prime}$ which is linear on edges. It is a morphism if and only if no edge $e$ 
of $T$ is mapped to a point. Suppose that $f(e)$ is a point $v \in T^{\prime}$. The group $G_{e}$ fixes $v$, and by (3) it fixes a non-degenerate segment $[v, w] \subset T^{\prime}$. We redefine $f$ on $e$ by subdividing $e$ into two halves and mapping each half onto $[v, w]$. Doing this equivariantly on each orbit of collapsed edges replaces the original $f$ by a morphism.

We have seen that (3) holds if $T$ and $T^{\prime}$ are reduced (Proposition 4.5).

Definition 4.7 (bi-morphism class). We say that two simplicial trees $T, T^{\prime}$ are $b i$ morphically equivalent, or belong to the same bi-morphism class, if they satisfy the equivalent conditions of Proposition 4.6. Metric trees are said to be bi-morphically equivalent if the underlying simplicial trees are.

A deformation space $\mathscr{D}$ is thus partitioned into bi-morphism classes. There is only one class if $\mathscr{D}$ is abelian, as any elliptic subgroup is bi-elliptic.

Definition 4.8 $\left(\mathcal{A}_{\text {min }}\right.$, reduced deformation space $\left.\mathcal{D}_{r}\right)$. Given $\mathcal{D}$, we denote by $\mathcal{A}_{\text {min }}$ the family of subgroups $H$ described in Proposition 4.5 (the bi-elliptic groups of any reduced $T$ ). They may be characterized as the groups which fix an edge in every tree belonging to $\mathscr{D}$. The associated restricted deformation space $\mathscr{D}_{\mathcal{A}_{\text {min }}} \subset \mathscr{D}$, consisting of trees $T \in \mathscr{D}$ with all edge stabilizers in $\mathcal{A}_{\text {min }}$, is the reduced deformation space, denoted $\mathscr{D}_{r}$.

The space $\mathscr{D}_{r}$ may be viewed as the bi-morphism class containing the reduced trees. Trees in $\mathscr{D}_{r}$ have the same generalized edge groups and the same bi-elliptic groups (those of $\mathcal{A}_{\text {min }}$ ), but not necessarily the same edge groups.

Proposition 4.2 provides a bound for the complexity of reduced trees. In general, there is no bound for complexity in $\mathcal{D}_{r}$ (it is infinite dimensional), unless some descending chain condition holds (for instance, if $\mathcal{A}_{\text {min }}$ consists of finite groups). This phenomenon already occurs in the canonical deformation space of GBS groups, for instance for BS $(2,4)$. There are also non-ascending GBS examples (see the end of Section 7).

Peripheral structure. Let $\mathcal{D}$ be a deformation space, and $\mathcal{A}_{\text {min }}$ as defined above. Let $\mathcal{D}_{r} \subset \mathcal{D}$ be the reduced deformation space, so that $T \in \mathscr{D}$ is in $\mathscr{D}_{r}$ if and only if all its edge stabilizers are in $\mathcal{A}_{\text {min }}$.

We say that a vertex stabilizer is big if it is not in $\mathcal{A}_{\min }$. We have seen (Proposition 4.3 and Corollary 4.4) that all trees in $\mathscr{D}_{r}$ have the same big vertex stabilizers, and that such vertex stabilizers coincide with maximal elliptic subgroups which are not in $\mathcal{A}_{\text {min }}$.

The goal of this subsection is to associate a peripheral structure $\mathcal{M}_{0}$ to a maximal elliptic subgroup $G_{0}$. It depends only on $\mathscr{D}$ and $G_{0}$.

First suppose that the maximal elliptic subgroup $G_{0}$ is big (not in $\mathcal{A}_{\text {min }}$ ). We consider the set of all subgroups $H \subset G_{0}$ which belong to $\mathcal{A}_{\text {min }}$. Among such 
groups, we define $H \leq K$ if $H$ is contained in a conjugate $g K^{-1}$ (with $g \in G_{0}$ ), and $H \sim K$ if $H \leq K$ and $K \leq H$.

Let $\mathcal{M}_{0}$ be the set of maximal elements of the set of equivalence classes. An element $m \in \mathcal{M}_{0}$ is a family of subgroups of $G_{0}$, stable under conjugation (when no bi-elliptic subgroup of $G_{0}$ is conjugate to a proper subgroup of itself, $m$ is just a conjugacy class of subgroups).

Proposition 4.9. Let $G_{0} \notin \mathcal{A}_{\min }$ be a maximal elliptic subgroup. Given $T \in \mathscr{D}_{r}$, let $v$ be the (unique) vertex with stabilizer $G_{0}$.

(1) If an edge $e$ is adjacent to $v$, there exists $m \in \mathcal{M}_{0}$ such that $G_{e}$ is contained in a group belonging to $m$.

(2) Given $m \in \mathcal{M}_{0}$, there exists an edge e adjacent to $v$ such that $G_{e}$ belongs to $m$.

(3) The image $\bar{v}$ of $v$ in the quotient graph $\Gamma=T / G$ has valence at least $\left|\mathcal{M}_{0}\right|$. In particular, $\mathcal{M}_{0}$ is finite.

(4) There exists $T^{\prime} \in D_{r}$ such that equality holds in (3): the valence of $\bar{v}$ is $\left|\mathcal{M}_{0}\right|$.

Proof. Let $\mathcal{C}_{0}$ consist of all groups $G_{f}$, for $f$ an edge of $T$ adjacent to $v$. It is a finite union of conjugacy classes of subgroups of $G_{0}$, and any bi-elliptic subgroup of $G_{0}$ is contained in an element of $\mathcal{C}_{0}$. Since $\mathcal{C}_{0} / \sim$ is finite, $G_{e}$ is contained in some $G_{f}$ which is maximal in $\mathcal{C}_{0} / \sim$. But $G_{f}$ is also maximal in the set of all bi-elliptic subgroups of $G_{0}$, so represents an element $m \in \mathcal{M}_{0}$. This proves (1).

To prove (2), let $H \subset G_{0}$ be a representative of $m \in \mathcal{M}_{0}$. Since $H$ is bi-elliptic, $H \subset G_{e}$ for some edge $e$ adjacent to $v$. By maximality, $H \sim G_{e}$.

Given $m^{\prime} \neq m$ in $\mathcal{M}_{0}$, define $H^{\prime}$ and $e^{\prime}$ similarly. The edges $e$ and $e^{\prime}$ are in different $G_{0}$-orbits, since otherwise $H \sim G_{e} \sim G_{e^{\prime}} \sim H^{\prime}$, contradicting $m \neq m^{\prime}$. Assertion (3) follows.

If equality does not hold in (3), there exist oriented edges $e, f$ with origin $v$, in distinct $G_{v}$-orbits, with $G_{e} \subset G_{f}$. Perform an elementary expansion, creating an edge $v w$ with stabilizer $G_{f}$, with $e$ and $f$ now attached to $w$. The new tree is again in $\mathscr{D}_{r}$ (no new bi-elliptic group is created), and the valence of $\bar{v}$ has decreased by 1 . Iterating this operation proves (4).

Definition 4.10 (peripheral structure $\mathcal{M}_{0}$ ). The set $\mathcal{M}_{0}$ is the peripheral structure of the maximal elliptic subgroup $G_{0} \notin \mathcal{A}_{\min }$. It describes stabilizers of edges adjacent to the vertex with stabilizer $G_{0}$, in any tree belonging to $\mathscr{D}_{r}$ (in particular, in reduced trees). When no bi-elliptic subgroup of $G_{0}$ is conjugate to a proper subgroup of itself, and equality holds in (3), the set $\mathcal{M}_{0}$ is simply the set of conjugacy classes of stabilizers of edges adjacent to $v$.

More generally, we now wish to consider any maximal elliptic subgroup $G_{0}$, whether in $\mathcal{A}_{\min }$ or not. The definition of $\mathcal{M}_{0}$ given above makes sense if $G_{0} \in \mathcal{A}_{\min }$, 
but is not satisfying since in this case $\mathcal{M}_{0}$ consists of a single equivalence class $m=\left\{G_{0}\right\}$. We shall give a better one.

Let $G_{0}$ be any maximal elliptic subgroup. Given a reduced $T \in \mathscr{D}$, choose $v$ with stabilizer $G_{0}$ (it is not necessarily unique). If $G_{e} \neq G_{v}$ for every adjacent edge $e=v w$, then $G_{0} \notin \mathcal{A}_{\min }$. Suppose therefore that there is $e=v w$ with $G_{e}=G_{0}$. Then $w$ is in the $G$-orbit of $v$ because $T$ is reduced, and $G_{w}=G_{0}$ because $G_{0}$ is maximal elliptic. The projection of the fixed subtree $T_{0}$ of $G_{0}$ into $\Gamma=T / G$ consists of loops attached to the projection $\bar{v}$. If $N\left(G_{0}\right)$ denotes the normalizer of $G_{0}$, the quotient $N\left(G_{0}\right) / G_{0}$ acts on $T_{0}$ freely, with one orbit of vertices. It is free, with rank $k$ equal to the number of loops.

To define $\mathcal{M}_{0}$, we consider all groups $H=G_{0} \cap G_{1}$, where $G_{1}$ is elliptic and not contained in $G_{0}$. Among such subgroups of $G_{0}$, we define $H \leq K$ whenever $H$ is contained in $g \mathrm{Kg}^{-1}$ for some element $g$ of the normalizer $N\left(G_{0}\right)$. The set $\mathcal{M}_{0}$ is then defined as above, as a set of maximal elements. This definition agrees with the one given before when $G_{0} \notin \mathcal{A}_{\min }$.

Proposition 4.11. Let $D$ be a deformation space, and $G_{0}$ be any maximal elliptic subgroup. Let $T \in \mathbb{D}$ be reduced. Let $v$ be any vertex with stabilizer $G_{0}$.

(1) If an edge e is adjacent to $v$ and $G_{e} \neq G_{0}$, there exists $m \in \mathcal{M}_{0}$ such that $G_{e}$ is contained in a group belonging to $m$.

(2) Given $m \in \mathcal{M}_{0}$, there exists an edge e adjacent to $v$ such that $G_{e}$ belongs to $m$.

(3) The image $\bar{v}$ of $v$ in the quotient graph $\Gamma=T / G$ has valence at least $\left|\mathcal{M}_{0}\right|+2 k$, where $k$ is the rank of the free group $N\left(G_{0}\right) / G_{0}$.

We leave the proof to the reader.

\section{Topologies on deformation spaces}

In this and the next section, $\mathscr{D}$ is a deformation space or, more generally, a restricted deformation space $\mathscr{D}_{\mathcal{A}}$. Because of Proposition 3.10 we assume that $\mathscr{D}$ is irreducible or genuine abelian. So far $\mathscr{D}$ was mostly viewed as a set. We shall now view $\mathscr{D}$ as a topological space and a complex.

We first consider the set $\mathcal{T}$ of all non-trivial minimal metric simplicial $G$-trees, up to equivariant isometry.

Three topologies. The equivariant Gromov-Hausdorff topology (or simply Gromov topology) on $\mathcal{T}$ is defined as follows. A fundamental system of neighborhoods for $T \in \mathcal{T}$ is given by sets $V_{T}(X, A, \varepsilon)$, with $X \subset T$ and $A \subset G$ finite sets and $\varepsilon>0$. By definition, $T^{\prime}$ is in $V_{T}(X, A, \varepsilon)$ if and only if there exists a "lifting" map $x \mapsto \tilde{x}$ from $X$ to $T^{\prime}$ such that $|d(x, g y)-d(\tilde{x}, g \tilde{y})|<\varepsilon$ for every $x, y \in X$ and $g \in A$. 
Remark 5.1. This topology may fail to be Hausdorff. For example, consider $T, T^{\prime}$ with the same length function, with $T$ a line and $T^{\prime}$ genuine abelian. Then every neighborhood of $T$ contains $T^{\prime}$.

The axes topology on $\mathcal{T}$ is the coarsest topology making each translation length function $T \mapsto \ell_{T}(g)$ continuous (it is coarser than the Gromov topology, as translation lengths are continuous in the Gromov topology). By [28], the two topologies have the same restriction to the set of non-abelian trees (note that dihedral trees are considered irreducible in [28]). Since non-abelian trees are determined by their length function, this restriction is Hausdorff.

We now discuss the weak topology. Beware that the open or closed cones to be defined now are not necessarily open or closed in the topological sense.

The set of metric $G$-trees obtained from a given simplicial tree $T$ by varying edgelengths (keeping them positive) will be called an open cone $\mathcal{C}$. Its dimension $p$ is the number of orbits of edges (recall that $T$ has no redundant vertices). Two metric trees are in the same open cone if and only if they are equivariantly homeomorphic.

If we allow edge-lengths of $T$ to become 0 (keeping at least one of them positive, so that the tree does not become trivial), we obtain the closed cone $\bar{\varphi}$ spanned by $T$. It consists of trees obtained from $T$ by (possibly non-elementary) collapses. It may be identified to an octant $\left(\mathbb{R}^{+}\right)^{p} \backslash\{(0, \ldots, 0)\}$. A closed cone is a finite union of open cones.

The weak topology on $\mathcal{T}$ is defined by declaring a set to be closed when its intersection with every closed cone is closed. It is always finer than the Gromov topology (a weakly converging sequence converges in the Gromov topology).

If a closed cone $\bar{e}$ meets an abelian deformation space $\mathscr{D}$, then it is contained in $\mathscr{D}$, as any collapse or expansion of an abelian tree is elementary (in the sense of Section 3). If $\mathscr{D}$ is irreducible, then $\mathscr{D} \cap \overline{\mathcal{C}}$ is not necessarily closed in $\bar{\ell}$ (this will be discussed later), but it is convex. To see this, we use Condition (7) of Theorem 3.8: if $\ell$ and $\ell^{\prime}$ are bi-Lipschitz equivalent, then any convex combination is also equivalent to $\ell$ and $\ell^{\prime}$.

Projectivized space. Rescaling the metric defines an action of the group of positive reals on $\mathcal{T}$, and the real object of interest is the quotient space $P \mathcal{T}$. It is a complex, an open (resp. closed) simplex being the projection of an open (resp. closed) cone of $\mathcal{T}$. The three topologies defined on $\mathcal{T}$ induce quotient topologies on $P \mathcal{T}$.

If we choose a finite generating system $S$ for $G$, the projection $\mathcal{T} \rightarrow P \mathcal{T}$ has a natural section defined as the set of trees such that $\sum_{s \in S} \ell(s)+\sum_{s, t \in S} \ell(s t)=1$ (recall that a tree such that all elements $s$ and $s t$ are elliptic is trivial [33]). This section is continuous in all three topologies (because the maps $T \mapsto \ell_{T}(g)$ are), so $\mathcal{T}$ is homeomorphic to $P \mathcal{T} \times \mathbb{R}$. In practice, we shall work in either $\mathcal{T}$ or $P \mathcal{T}$, and the results will automatically apply to the other space. 
Topologies on $\mathscr{D}$. We now restrict these topologies to a given deformation space $\mathscr{D}$ (irreducible or genuine abelian). First consider the Gromov topology and the axes topology.

If $\mathscr{D}$ is irreducible, the restrictions are the same. The induced topology will be called the Gromov topology on $\mathscr{D}$. It is Hausdorff.

If $\mathscr{D}$ is genuine abelian, all trees in $\mathscr{D}$ have the same length function (up to scaling) and the axes topology is trivial on $P \mathscr{D}$. We therefore only consider the Gromov topology. We shall prove that it is Hausdorff (it is not obvious in this case), see Proposition 5.7.

We now consider the weak topology. There is a subtlety here in the irreducible case due to the fact that $\mathcal{D}$, though a union of open cones of $\mathcal{T}$, is not necessarily a union of closed cones ( $\mathscr{D}$ is not closed in $\mathcal{T}$ for the weak topology). Indeed, the closed cone $\bar{\ell}$ of $\mathcal{T}$ spanned by a given $T \in \mathscr{D}$ is not always entirely contained in $\mathscr{D}$, as collapsing certain orbits of edges may yield a tree not in $\mathscr{D}$. The intersection of $\bar{C}$ with $\mathscr{D}$ is the set of trees that may be obtained from trees in $\mathcal{C}$ by elementary collapses (as defined in Section 3). It will be called a closed cone of $\mathscr{D}$ (it may have faces "at infinity"). Closed simplices of $P D$ are defined similarly. A closed cone (resp. closed simplex) is a finite union of open cones (resp. simplices). It always contains reduced trees.

Remark. Although simplices of $P D$ are not actual simplices, there is a standard way to obtain a genuine simplicial complex (see [12], [26]). Start from the barycentric subdivision of $P \mathcal{T}$ and look at the union of closed simplices which are contained in $P \mathscr{D}$. One easily checks that one obtains a simplicial complex contained in $P \mathscr{D}$ on which $P \mathscr{D}$ retracts by deformation.

The weak topology on $\mathscr{D}$ is defined as the induced topology. A subset of $\mathscr{D}$ is closed in $\mathscr{D}$ if and only if its intersection with any closed cone of $\mathscr{D}$ is closed (in the cone). Similarly, a subset of $P D$ is closed in the weak topology if and only if its intersection with any closed simplex of $P \mathscr{D}$ is closed.

As pointed out in [26], the weak topology does not always coincide with the Gromov topology or the axes topology. Indeed, the weak topology on a locally infinite complex is never metrizable (there is no countable basis of neighborhoods).

Proposition 5.2. Let $D$ be a deformation space. The Gromov topology and the weak topology induce the same topology on any finite union of cones of $D$ (resp. of simplices of $P D$ ).

The proof will show that the analogous result for cones in $\mathcal{T}$ is true if none of the cones is contained in an abelian deformation space. But it may be false on the union of two cones contained in different abelian deformation spaces (see Remark 5.1). The proof of the proposition for $\mathscr{D}$ abelian requires the fact that $\mathscr{D}$ is Hausdorff in the Gromov topology. This will be proved at the end of this section. 
Proof. It suffices to show the result in $P \mathscr{D}$. Let $\Sigma$ be any finite union of simplices of $P \mathcal{T}$ (not necessarily contained in $P D$ ). Let $\bar{\Sigma}$ be its weak closure in $P \mathcal{T}$. Trees in $\bar{\Sigma}$ are obtained by performing (possibly non-elementary) collapses on trees in $\Sigma$. Being a finite union of closed simplices of $P \mathcal{T}$, the space $\bar{\Sigma}$ is weakly compact. Since the weak topology is finer than the Gromov topology, it is enough to show that $\bar{\Sigma}$ is Hausdorff in the Gromov topology.

If $\Sigma$ contains only non-abelian trees, so does $\bar{\Sigma}$, and we know that the set of nonabelian trees is Hausdorff in the Gromov topology. If $\Sigma$ is contained in a genuine abelian deformation space $\mathscr{D}$, we have seen that $\bar{\Sigma}=\Sigma$. We then use the fact that $P \mathscr{D}$ is Hausdorff in the Gromov topology (see Proposition 5.7).

Remark 5.3. In particular, the topologies agree on any open or closed cone of $\mathscr{D}$. Such a cone being convex (as a subset of $\mathbb{R}^{p}$ ), it is contractible. The same holds for simplices of $P \mathscr{D}$.

Locally finite trees. In this subsection we assume that all trees in $\mathscr{D}$ are locally finite (see Section 4 for a discussion, and examples of local finiteness). In this case, it is easy to show that the complex $P \mathscr{D}$ is locally finite (see [25]). Moreover, all vertex and edge stabilizers are commensurable, so they are all finitely generated, or all infinitely generated. We shall show:

Proposition 5.4. If $D$ consists of locally finite trees with finitely generated vertex stabilizers, then the Gromov topology and the weak topology coincide on $\mathscr{D}$ and PD.

Let $T$ be a tree. Denote by $E(T)$ the set of oriented open edges of $T$, and by $e \mapsto \bar{e}$ the involution mapping an edge to the opposite edge. Say that three oriented edges $e_{1}, e_{2}, e_{3}$ are aligned in this order if there is a geodesic going through $e_{1}, e_{2}$ and $e_{3}$ successively, with the correct orientation.

Lemma 5.5. Let $T, T^{\prime}$ be two simplicial $G$-trees. Then $T$ is a (maybenon-elementary) collapse of $T^{\prime}$ if and only if there exists a $G$-equivariant injection $\varphi: E(T) \hookrightarrow E\left(T^{\prime}\right)$, with $\varphi(\bar{e})=\overline{\varphi(e)}$, which preserves alignment in the following sense: if $e_{1}, e_{2}, e_{3}$ are aligned in this order, then so are $\varphi\left(e_{1}\right), \varphi\left(e_{2}\right), \varphi\left(e_{3}\right)$.

Proof. If $f: T^{\prime} \rightarrow T$ is a collapse map, then the preimage of each open edge of $T$ is an open edge of $T^{\prime}$. This defines a map $\varphi: E(T) \rightarrow E\left(T^{\prime}\right)$ with the desired properties.

Conversely, we are given $\varphi$ and we want to define a collapse map $f: T^{\prime} \rightarrow T$. Let $I \subset T^{\prime}$ be the union of all open edges in the image of $\varphi$. We first define $f$ on $I$, using $\varphi^{-1}$. Next, consider $x \in T^{\prime} \backslash I$. Let $\alpha, \beta$ be two edges in $I$ such that $x$ is in the convex hull of $\alpha \cup \beta$ (they exist because $T^{\prime}$ is minimal). Choose $\alpha, \beta$ so that this 
hull is minimal. Orient $\alpha$ and $\beta$ so that they point towards $x$. Denote by $y_{\alpha}$ and $y_{\beta}$ the terminal points of $\varphi^{-1}(\alpha)$ and $\varphi^{-1}(\beta)$ respectively. If $y_{\alpha} \neq y_{\beta}$, there is an edge $e$ between them, and $e \neq \alpha, \beta$ because $\varphi$ preserves alignment. Then $\varphi(e)$ is between $\alpha$ and $\beta$, contradicting minimality in the choice of $\alpha, \beta$. Thus $y_{\alpha}=y_{\beta}$, and we may define $f(x)=y_{\alpha}$ consistently. The map $f$ is clearly a collapse map.

Proof of Proposition 5.4. If $T, T^{\prime}$ are metric trees, we say that $T^{\prime}$ collapses to $T$ if this is true for the underlying simplicial trees. Local finiteness implies that, given $T \in \mathscr{D}$, the set $S(T)$ consisting of trees of $\mathscr{D}$ which collapse to $T$ is a finite union of cones. Since the weak topology is finer than the Gromov topology and both topologies coincide on $S(T)$ by Proposition 5.2, it suffices to prove that $S(T)$ contains the intersection of $\mathscr{D}$ with some Gromov-neighborhood $V_{T}(X, A, \varepsilon)$ of $T$.

By local finiteness of $T$, one can find a finite subtree $K$ of $T$ such that, for each pair $\alpha, \beta$ of adjacent edges, there exists $g \in G$ mapping both $\alpha$ and $\beta$ into $K$. Let $X$ be the set of vertices of $K$. Let $A \subset G$ be a finite set containing a set of generators of each $G_{v}$ for $v \in X$ (recall that each $G_{v}$ is assumed to be finitely generated) and also an element sending $v_{1}$ to $v_{2}$ for each pair of points $v_{1}, v_{2} \in X$ lying in the same $G$-orbit. Take $\varepsilon$ very small compared to the lengths of edges of $T$. We show that any $T^{\prime} \in \mathscr{D} \cap V_{T}(X, A, \varepsilon)$ collapses to $T$.

We first construct an equivariant map $v \mapsto v^{\prime}$ from $V(T)$ (the set of vertices of $T$ ) to $T^{\prime}$. If $v \in X$ we denote by $\tilde{v}$ a "lift" of $v$ to $T^{\prime}$, as in the definition of $V_{T}(X, A, \varepsilon)$. For each $G$-orbit of vertices, choose a representative $v_{i} \in X$. Since $G_{v_{i}}$ is elliptic in $T^{\prime}$, and the generators of $G_{v_{i}}$ move $\tilde{v}_{i}$ by less than $\varepsilon$, the group $G_{v_{i}}$ has a fixed point in $T^{\prime}$ at distance at most $\varepsilon / 2$ from $\tilde{v}_{i}$. We define $v_{i}^{\prime}$ to be such a point, and we extend equivariantly.

If $v \in X$ is not a $v_{i}$, the point $v^{\prime}$ is at distance at most $3 \varepsilon / 2$ of $\tilde{v}$. It follows that, if $u v$ and $u w$ are two adjacent edges of $T$, then the distances between $u^{\prime}, v^{\prime}, w^{\prime}$ are $4 \varepsilon$-close to the distances between $u, v, w$ (this is true if the points are vertices of $K$, hence in general by our choice of $K$ ). In particular, the overlap between $u^{\prime} v^{\prime}$ and $u^{\prime} w^{\prime}$ has length at most $6 \varepsilon$.

We shall now define a map $\varphi: E(T) \rightarrow E\left(T^{\prime}\right)$ as in Lemma 5.5. For each edge $e=u v$ of $T$, let $I_{e}$ be the segment of $T^{\prime}$ obtained by removing from $u^{\prime} v^{\prime}$ the $6 \varepsilon$-balls centered at $u^{\prime}$ and $v^{\prime}$. Our choice of $\varepsilon$ guarantees that $I_{e}$ has length much bigger than $\varepsilon$. If $e_{1}, e_{2}$ are adjacent, then $I_{e_{1}} \cap I_{e_{2}}$ is empty and $d\left(I_{e_{1}}, I_{e_{2}}\right) \leq 12 \varepsilon$. It follows that, given a geodesic edge path $e_{1}, \ldots, e_{n}$ in $T$, the segments $I_{e_{1}}, \ldots, I_{e_{n}}$ are aligned in this order in $T^{\prime}$. In particular, $I_{e_{1}} \cap I_{e_{2}}=\emptyset$ for any pair of edges $e_{1} \neq e_{2}$,

Moreover, no $I_{e}$ contains a vertex in its interior. Otherwise, the subtree branching from $I_{e}$ would contain a segment $I_{e^{\prime}}$ (by minimality). We would get a contradiction by considering the edge path from $e$ to $e^{\prime}$ in $T$ and the corresponding aligned segments in $T^{\prime}$.

Thus, one can define $\varphi: E(T) \hookrightarrow E\left(T^{\prime}\right)$ by mapping an edge $e$ to the edge of $T^{\prime}$ containing $I_{e}$. By the lemma, $T$ is a collapse of $T^{\prime}$. 
The closure of an irreducible space. Let $\mathscr{D}$ be an irreducible deformation space. We consider its closure $\bar{D}$ in the space $\widetilde{T}_{\mathbb{R}}$ of non-trivial minimal $\mathbb{R}$-trees, equipped with the Gromov topology. It usually contains non-simplicial $\mathbb{R}$-trees (see [13] for a concrete example).

If $T \in \bar{D}$ we denote by $\ell$ its length function. Given $g \in G$, we denote by $A_{g}$ the axis of $g$ if $g$ is hyperbolic in $T$, its fixed point set if it is elliptic (an element which is hyperbolic in $\mathscr{D}$ may become elliptic in $T \in \bar{D}$ ).

Proposition 5.6. Let $\mathfrak{D}$ be an irreducible deformation space. Let $\bar{D}$ be its closure in the Gromov topology. Let $T$ be an $\mathbb{R}$-tree in $\bar{D}$.

(1) If $g \in G$ is hyperbolic in $\mathscr{D}$ but elliptic in $T$, its fixed point set contains no tripod.

(2) If $g, h,[g, h]$ are hyperbolic in $\mathscr{D}$, then $A_{g} \cap A_{h}$ is a segment (possibly empty or degenerate) whose length is at most $\ell(g)+\ell(h)$.

(3) $T$ is irreducible.

(4) $\bar{D}$ is also the closure of $\mathscr{D}$ in the axes topology.

Proof. Assertion (1) holds because fixing a tripod is an open condition (see [29], p. 153). To prove (2), note that $A_{g}$ and $A_{h}$ contain no tripods. If $\left|A_{g} \cap A_{h}\right|>$ $\ell(g)+\ell(h)$, this inequality holds in trees $T^{\prime} \in \mathscr{D}$ close enough to $T$ (if $x \in A_{g}$ any lift $\tilde{x}$ of $x$ to $T^{\prime}$ is close to the axis of $g$ in $\left.T^{\prime}\right)$. A standard argument shows that $[g, h]$ is elliptic in $T^{\prime}$, a contradiction.

Since $\mathscr{D}$ is irreducible, we can find $g, h$ as in (2). Compactness of $A_{g} \cap A_{h}$ implies that $T$ is not abelian. Applying the argument to $g^{2}$ and $h^{2}$ shows that $T$ is not dihedral, so it is irreducible.

Recall that the Gromov topology and the axes topology agree on the set of nonabelian trees [28]. To prove (4), it suffices to see that length functions of trees in $\mathscr{D}$ cannot accumulate onto an abelian length function $|\varphi|$, with $\varphi: G \rightarrow \mathbb{R}$ a non-trivial homomorphism.

Supposing they do, fix $g \in G$ with $\varphi(g)>0$. It is hyperbolic in $\mathscr{D}$. Since $\mathscr{D}$ is irreducible, we can find a conjugate $h$ of $g$ such that $[g, h]$ is hyperbolic in $\mathscr{D}$ (choose $h$ such that $g$ and $h$ have disjoint axes in some tree of $\mathscr{D}$ ). Now consider $T \in \mathscr{D}$ whose length function $\ell$ is close to $|\varphi|$ when applied to $g, h$, and $g^{3} h^{-3}$ (in particular, $\ell\left(g^{3} h^{-3}\right)$ is small). Using the formulas in [28], Proposition 1.6, one sees that the intersection of the axes of $g$ and $h$ in $T$ must contain a segment of length almost $3 \varphi(g)$. Since $3 \varphi(g)>2 \varphi(g) \sim \ell(g)+\ell(h)$, the commutator $[g, h]$ is elliptic in $T$, a contradiction. 


\section{Abelian deformation spaces are Hausdorff}

Proposition 5.7. Any genuine abelian deformation space $\mathscr{D}$ is Hausdorff in the Gromov topology.

Proof. If $T \in \mathscr{D}$, all translation axes $A_{g}$ in $T$ have a common end, which is fixed by $G$. We say that a hyperbolic $g$ is positive (in $T$ ) if it moves points away from the fixed end, negative if it moves towards the end (in other words, we think of the fixed end as being at $-\infty$ ).

It is easy to check that a hyperbolic element $g$ is positive if and only if, given any $h \in G$, the group generated by the commutators $\left[g^{n}, h\right]$, with $n>0$, is elliptic. In particular, positivity does not depend on the choice of $T$ in $\mathscr{D}$.

Similarly, the fact that two hyperbolic elements $g, h$ have the same axis depends only on $\mathscr{D}$ (it is characterized by ellipticity of the group generated by the elements $\left.\left[g^{n}, h\right], n \in \mathbb{Z}\right)$.

Consider positive hyperbolic elements $g, h$ with different axes. Let $P_{g h}$ be the endpoint of the ray $A_{g} \cap A_{h}$ (see Figure 1). It is characterized by the equations

$$
\begin{aligned}
d(P, g P) & =\ell(g), \\
d(P, h P) & =\ell(h), \\
d(g P, h P) & =\ell(g)+\ell(h) .
\end{aligned}
$$

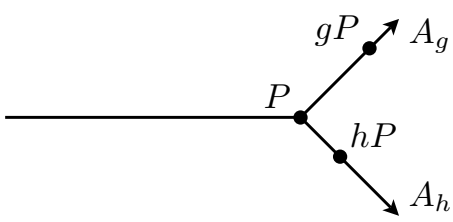

Figure 1. Characterizing the endpoint of $A_{g} \cap A_{h}$.

Furthermore, this system is stable in the following sense: there exists a universal constant $C$ such that if $P$ satisfies each equation up to $\varepsilon$, then it is $C \varepsilon$-close to $P_{g h}$.

Now consider three positive hyperbolic elements $g, h, i$ with distinct axes. We claim that the number $\delta_{T}=d\left(P_{g h}, P_{g i}\right)$ depends continuously on $T$ (in the Gromov topology).

If $T^{\prime}$ is another tree in $\mathscr{D}$ with length function $\ell^{\prime}$, we denote by $\left(1_{g h}^{\prime}\right)$ the system $\left(1_{g h}\right)$ with $\ell$ replaced by $\ell^{\prime}$. Note that the right-hand sides depend continuously on $T$. Fix $\varepsilon>0$. If $T^{\prime}$ is close enough to $T$, we can find lifts $\widetilde{P}_{g h} \in T^{\prime}$ satisfying $\left(1_{g h}^{\prime}\right)$ up to $\varepsilon$, and $\widetilde{P}_{g i}$ satisfying $\left(1_{g i}^{\prime}\right)$ up to $\varepsilon$, with $d\left(\widetilde{P}_{g h}, \widetilde{P}_{g i}\right) \varepsilon$-close to $\delta_{T}$. We get $\left|\delta_{T^{\prime}}-\delta_{T}\right| \leq(2 C+1) \varepsilon$, showing continuity. 
To prove that $\mathcal{D}$ is Hausdorff, suppose that $T, T^{\prime}$ do not have disjoint neighborhoods. Then $\delta_{T^{\prime}}=\delta_{T}$ for all ordered triples $(g, h, i)$ as above. We show that this implies $T=T^{\prime}$.

Denote by $g_{1}, g_{2}, \ldots$ the positive hyperbolic elements of $\mathcal{D}$. Write $A_{i}$ (resp. $A_{i}^{\prime}$ ) for the axis of $g_{i}$ in $T$ (resp. $T^{\prime}$ ). We may assume that $A_{1} \neq A_{2}$. Since $A_{i}=A_{j}$ is equivalent to $A_{i}^{\prime}=A_{j}^{\prime}$, we get $A_{1}^{\prime} \neq A_{2}^{\prime}$.

There is a unique isometry $f_{2}: A_{1} \cup A_{2} \rightarrow A_{1}^{\prime} \cup A_{2}^{\prime}$ sending $A_{1}$ to $A_{1}^{\prime}$ and $A_{2}$ to $A_{2}^{\prime}$. If $A_{3}$ is distinct from $A_{1}$ and $A_{2}$, its position with respect to $A_{1} \cup A_{2}$ is completely determined by the three numbers $\delta_{T}$ associated to the triples $\left(g_{1}, g_{2}, g_{3}\right)$, $\left(g_{2}, g_{3}, g_{1}\right),\left(g_{3}, g_{1}, g_{2}\right)$ (one of these numbers is 0 , the other two are equal). Thus $f_{2}$ extends uniquely to an isometry $f_{3}: A_{1} \cup A_{2} \cup A_{3} \rightarrow A_{1}^{\prime} \cup A_{2}^{\prime} \cup A_{3}^{\prime}$ sending $A_{3}$ to $A_{3}^{\prime}$.

By a similar argument, $f_{3}$ has a sequence of successive unique extensions $f_{n}: A_{1} \cup \cdots \cup A_{n} \rightarrow A_{1}^{\prime} \cup \cdots \cup A_{n}^{\prime}$ sending $A_{i}$ to $A_{i}^{\prime}$ for $i \leq n$, and we finally get a global isometry $f: T \rightarrow T^{\prime}$ sending every $A_{g}$ to $A_{g}^{\prime}$. By uniqueness, $f$ is $G$-equivariant.

\section{Contractibility}

Let $\mathcal{D}$ be a deformation space, or more generally, a restricted deformation space $\mathscr{D}_{\mathcal{A}}$. We shall prove:

Theorem 6.1. Let $\mathcal{D}$ be a deformation space.

(1) $\mathcal{D}$ is contractible in the weak topology.

(2) If $\mathscr{D}$ contains a tree $T_{0}$ with finitely generated vertex stabilizers, then $\mathscr{D}$ is contractible in the Gromov topology.

(3) If $D$ is irreducible and contains a tree $T_{0}$ with finitely generated vertex stabilizers, then its closure $\bar{D}$ in the Gromov topology is contractible.

The same results hold for $P \mathcal{D}$.

Recall that $\bar{D}$ is the closure of $\mathscr{D}$ in $\mathcal{T}_{\mathbb{R}}$ (the space of non-trivial minimal $\mathbb{R}$-trees, equipped with the Gromov topology or the axes topology, see Proposition 5.6). Its projectivization is the closure of $P \mathscr{D}$ in $P \mathcal{T}_{\mathbb{R}}$. Note that the closure in $\mathcal{T}$ (equipped with the Gromov topology) of a genuine abelian space is never Hausdorff, since adding the linear abelian tree having the same length function makes $\mathscr{D}$ non-Hausdorff (see Remark 5.1).

The key technique for proving contractibility is Skora's idea of deforming morphisms between metric trees: given any morphism $f: S_{0} \rightarrow S$, there is a canonical way of constructing intermediate trees $S_{t}$, for $0 \leq t \leq \infty$, with $S_{\infty}=S$ (see [7], [20], [35]). The tree $S_{t}$ depends continuously on $f$ and $t$ in the Gromov topology. It 
belongs to $\mathcal{D}\left(\right.$ resp. $\mathscr{D}_{\mathcal{A}}$ ) if both $S_{0}$ and $S$ do, for there are morphisms $\varphi_{t}: S_{0} \rightarrow S_{t}$ and $\psi_{t}: S_{t} \rightarrow S$ with $\psi_{t} \circ \varphi_{t}=f$.

Defining a contraction. In this subsection, we explain how to define a map $\rho: \mathscr{D} \times[0,+\infty] \rightarrow \mathscr{D}$ with $\rho(T, \infty)=T$ and $\rho(\mathscr{D} \times\{0\})$ contained in a contractible subset. We shall then show Assertions (1) and (2) by establishing continuity of $\rho$. The proof of (3) will be given at the end of the section.

Fix $T_{0} \in \mathcal{D}$, with finitely generated vertex stabilizers if proving (2). We may assume that it is reduced (elementary collapses do not create new vertex stabilizers). We view $T_{0}$ as a simplicial tree, and we let $\ell_{0} \subset \mathscr{D}$ be the open cone containing it (it is the set of all metric trees with $T_{0}$ as underlying simplicial tree). Recall (Remark 5.3) that $\ell_{0}$ is contractible, both in the Gromov topology and in the weak topology.

We shall associate to $T \in \mathcal{D}$ a morphism $f_{T}: T_{0}(T) \rightarrow T$, where $T_{0}(T)$ is a metric tree belonging to $\ell_{0}$. Skora's deformation (see above) provides intermediate trees $T_{t}(T)$. Setting $\rho(T, t)=T_{t}(T)$ yields a map $\rho: \mathcal{D} \times[0,+\infty] \rightarrow \mathcal{D}$, with $\rho(T, 0)=T_{0}(T)$ and $\rho(T, \infty)=T$ (if some intermediate tree fails to be minimal, we replace it by its minimal subtree). Since $T_{0}(T)$ belongs to the contractible set $\mathcal{C}_{0}$, we deduce that $\mathscr{D}$ is contractible (assuming continuity of $\rho$ ).

There remains to construct $f_{T}$. It is easy in the following special case (see [20]). Suppose that $\mathcal{D}$ is a restricted deformation space $\mathscr{D}_{\mathcal{A}}$, and no vertex stabilizer $G_{v}$ of $T_{0}$ belongs to $\mathcal{A}$. Then $G_{v}$ fixes a unique point $v^{\prime}$ in $T$, and $f_{T}$ is defined on the vertex set of $T_{0}$ by sending $v$ to $v^{\prime}$. Since $T_{0}$ is reduced, adjacent vertices are mapped to distinct points by Remark 3.7. There is a unique edge-length assignment on $T_{0}$ such that the natural extension of $f_{T}$ is an isometry when restricted to edges. This assignment defines the metric tree $T_{0}(T) \in \mathcal{C}_{0}$, and $f_{T}: T_{0}(T) \rightarrow T$ is a morphism.

In general (as in Skora's proof that Culler-Vogtmann space is contractible), constructing $f_{T}$ requires choosing a basepoint $P \in T$ (continuously). Once $P$ has been chosen, one obtains $f_{T}$ as follows. Fix a representative $v_{j} \in T_{0}$ in each orbit of vertices and define $f_{T}\left(v_{j}\right) \in T$ as the projection $P_{j}$ of the basepoint $P$ onto the fixed point set of $G_{v_{j}}$. Then extend $f_{T}$ equivariantly to the vertex set of $T_{0}$ (this is possible because $G_{v_{j}}$ fixes $P_{j}$ ), and to the edges as before.

Skora's construction of a basepoint was based on minimization. We give a different one.

First suppose that $\mathcal{D}$ is irreducible. Fix elements $g, h \in G$ such that $g, h$ and $[g, h]$ are hyperbolic in $\mathcal{D}$. If $T \in \mathscr{D}$ the axes $A_{g}$ and $A_{h}$ of $g$ and $h$ in $T$ have compact intersection (see Proposition 5.6), and we use this fact to define $P$.

The most symmetric way would be to define $P$ as the midpoint of the segment $A_{g} \cap A_{h}$ if $A_{g}$ and $A_{h}$ meet, and as the midpoint of the "bridge" between them if they are disjoint. This "symmetric basepoint" will be used at the end of this section (see Figure 4), but for technical reasons (in the proof of Corollary 6.3) we now define $P$ in a slightly different way (see Figure 2 later in this section). If $A_{g}$ and $A_{h}$ meet, we 
order $A_{g}$ so that the action of $g$ is by a positive translation and we let $P$ be the largest element of the segment $A_{g} \cap A_{h}$. If $A_{g}$ and $A_{h}$ are disjoint, we define $P$ as the point of $A_{g}$ closest to $A_{h}$.

If $\mathscr{D}$ is abelian, we fix two positive hyperbolic elements $g, h \in G$ with distinct axes in some and therefore in any $T \in \mathscr{D}$ (see the proof of Proposition 5.7). We define $P$ as the endpoint of the ray $A_{g} \cup A_{h}$.

This completes the definition of the map $\rho: \mathscr{D} \times[0,+\infty] \rightarrow \mathscr{D}$. We now study its topological properties.

Lemma 6.2. Suppose that, given any two vertices $v, w$ of $T_{0}$, the map $T \mapsto$ $d\left(f_{T}(v), f_{T}(w)\right)$ from $\mathbb{D}$ equipped with the Gromov topology to $\mathbb{R}$ is continuous. Then the map $\rho$ is continuous in the Gromov topology.

Recall that all trees $T_{0}(T)$ have $T_{0}$ as their underlying simplicial tree, so their vertex sets are canonically identified.

Proof. If the condition of the lemma is satisfied, it is easy to see that the morphism $f_{T}$ depends continuously on $T$ (there is a natural Gromov topology on the space of morphisms, see e.g. [20]). Continuity of $\rho$ then follows from continuity of Skora's deformation (see [20], Section 3). We may have to replace a tree by its minimal subtree, but this is a continuous operation in the Gromov topology.

Corollary 6.3. If 8 is a closed cone of $\mathscr{D}$, the restriction $\rho_{8}: \& \times[0, \infty] \rightarrow \mathscr{D}$ is continuous in the Gromov topology.

Proof. By Proposition 5.2, the Gromov topology and the weak topology agree on 8 . It is homeomorphic to a Euclidean cone, parametrized by edge-lengths $\ell_{1}, \ldots, \ell_{p}$ ( $p$ is the number of orbits of edges of trees in the interior of 8 ; as discussed in Section 5, the cone may fail to be closed as a Euclidean cone). The basepoint $P$, and its projections $P_{j}$, were defined using only the simplicial structure of $T$, with no reference to edge-lengths. In restriction to $\delta$, any function $d\left(f_{T}(v), f_{T}(w)\right)$ as in Lemma 6.2 is thus a fixed linear combination of $\ell_{1}, \ldots, \ell_{p}$, hence is continuous.

Contractibility in the weak topology. We now show that $\rho$ is continuous in the weak topology, thus proving contractibility of $\mathscr{D}$ in that topology. By definition of the weak topology, it suffices to prove continuity of restrictions $\rho_{s}: \delta \times[0, \infty] \rightarrow \mathscr{D}$, with the target space equipped with the weak topology.

As in [20], we show this by proving that when $T$ varies inside 8 , the set of intermediate trees $T_{t}(T)=\rho(T, t)$ only meets finitely many cones.

Lemma 6.4 (finiteness lemma). Let $\mathcal{C}$ be an open cone in $D$. The set of intermediate trees $T_{t}(T)=\rho(T, t)$, for $t \geq 0$ and $T \in \mathcal{C}$, is contained in a finite union of cones. 
The meaning of this lemma is that there are only finitely many possibilities for $T_{t}(T)$ as a non-metric tree, as $t$ and $T$ vary. The lemma has weaker hypotheses than Lemma 4.3 of [20], and the conclusion is weaker: we do not control all trees $T^{\prime}$ such that $f_{T}$ factors through $T^{\prime}$, only those which occur as intermediate trees in the deformation.

Since the closed cone $\delta$ is a finite union of open cones, contractibility of $\mathscr{D}$ in the weak topology follows from the finiteness lemma together with Corollary 6.3 and Proposition 5.2.

The proof of Lemma 6.4 is rather complicated, so we first prove the following simpler result:

Lemma 6.5. Let $f: T_{0} \rightarrow T$ be a morphism between two metric trees belonging to the same deformation space. The set of intermediate trees $T_{t}$ is contained in a finite union of cones.

Proof. The proof we give is not the shortest possible, but the method will be used to prove Lemma 6.4. We first recall how the intermediate trees $T_{t}$ and the factorization of $f$ through morphisms $\varphi_{t}: T_{0} \rightarrow T_{t}$ and $\psi_{t}: T_{t} \rightarrow T$ are constructed (see [20], [35]).

Two points $x, y \in T_{0}$ with $f(x)=f(y)$ are identified in $T_{t}$ if and only if the image of the segment $[x, y]$ by $f$ is contained in the $t$-ball centered at $f(x)$. This defines $T_{t}$ as a set. The distance function on $T_{t}$ is the maximal one making the quotient map $\varphi_{t}$ 1-Lipschitz, and $\psi_{t}$ is defined in the obvious way. See [20] for details.

A key feature of this construction is that the geometry of the image of a segment $[x, y]$ in $T_{t}$ depends only on $t$ and the restriction of $f$ to $[x, y]$.

By subdividing $T_{0}$ and $T$ we may assume that $f$ is a simplicial map (an edge is mapped to an edge). Choose a set of representatives $\varepsilon_{i}$ for orbits of non-oriented edges of $T$. Label edges of $T$ by the corresponding $i$. Also orient edges of $T$ in an equivariant way. Use $f$ to lift labeling and orientation to edges of $T_{0}$.

Consider a couple $(\alpha, \beta)$ consisting of distinct non-oriented edges of $T_{0}$ with the same image in $T$. Let $E$ be the convex hull of $\alpha \cup \beta$ (a segment). Since $T_{0}$ and $T$ have the same elliptic subgroups, Remark 3.9 implies that there is a bound for the length of $E$ (as $(\alpha, \beta)$ varies).

The morphism $\varphi_{t}: T_{0} \rightarrow T_{t}$ folds $E$ into a finite subtree $E(t)$ which interpolates between $E$ and its image $F=f(E)$ in $T$. As already pointed out, this folding process depends only on the restriction of $f$ to $E$. We formalize this observation as follows.

Let $(\alpha, \beta)$ and $\left(\alpha^{\prime}, \beta^{\prime}\right)$ be two couples as above. We say that they are equivalent if there exists a commutative diagram

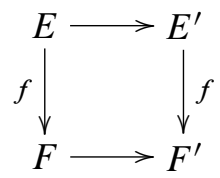


where the horizontal arrows are label-preserving and orientation-preserving simplicial isomorphisms. In particular, $(g . \alpha, g . \beta)$ is equivalent to $(\alpha, \beta)$ for any $g \in G$. Also note that the common label of $\alpha$ and $\beta$ gives a well-defined label $i$ for the corresponding equivalence class.

Equivalence implies that the same folding takes place in $E$ as in $E^{\prime}$ as $t$ increases; in particular, $E(t)$ and $E^{\prime}(t)$ are isometric. Furthermore, there are only finitely many equivalence classes since $E$ has bounded length and there are finitely many labels.

Given $t>0$ and an equivalence class $c$, we shall define a (possibly empty or degenerate) subsegment $I_{c}(t)$ of the edge $\varepsilon_{i}$ (where $i$ is the label of $c$ ). It will encode the way in which folding occurs in $T_{t}$.

Represent $c$ by $(\alpha, \beta)$, with $\alpha, \beta$ mapping onto $\varepsilon_{i}$. The edges $\alpha, \beta$ are mapped isometrically into $T_{t}$, but their images may be glued along a closed segment (possibly a point). We define $I_{c}(t)$ as the image of this segment in $\varepsilon_{i}$ (it is empty if no gluing occurs). It depends only on $c$, not on the choice of $(\alpha, \beta)$.

We have associated to $t$ a finite family of subsegments $I_{c}(t)$, one for each equivalence class $c$. We focus on the combinatorial structure of this family: we say that $t$ and $t^{\prime}$ are similar if, for every $i$, there exists an orientation-preserving homeomorphism of $\varepsilon_{i}$ mapping each $I_{c}(t)$ to $I_{c}\left(t^{\prime}\right)$. There are only finitely many similarity classes. We complete the proof by fixing a class and showing that, as a non-metric tree, $T_{t}$ does not depend on the choice of $t$ in that class.

Subdivide each $\varepsilon_{i}$ by adding all endpoints of segments $I_{c}$. Extend the subdivision equivariantly, and lift it to $T_{0}$ and $T_{t}$ using $f$ and $\psi_{t}$. We use the symbol ${ }^{\sim}$ to indicate the subdivided trees. The simplicial structure on $\widetilde{T}_{0}$ is fixed, and the map $\varphi_{t}$ is now simplicial (it sends edges to edges). The simplicial structure on $\widetilde{T}_{t}$ is completely determined by knowing which pairs of edges of $\widetilde{T}_{0}$ get identified in $\widetilde{T}_{t}$ (see [20]).

So consider edges $\widetilde{\alpha}, \widetilde{\beta}$ with the same image $\tilde{\varepsilon}$ in $\widetilde{T}$. They are contained in edges $\alpha, \beta$ of $T_{0}$ (the unsubdivided tree). Using $G$-equivariance, we may assume that $\alpha$ and $\beta$ map onto some $\varepsilon_{i}$. Now observe that $\widetilde{\alpha}$ and $\widetilde{\beta}$ are identified in $\widetilde{T}_{t}$ if and only if $\tilde{\varepsilon}$ is contained in $I_{c}(t)$, where $c$ is the equivalence class of $(\alpha, \beta)$. This description does not involve $t$ (in the given similarity class), so $\widetilde{T}_{t}$ depends only on the similarity class (as a simplicial tree).

Proof of Lemma 6.4. Choosing a point in $\mathcal{C}$ amounts to choosing a metric $m$ (which assigns lengths to edges) on a fixed non-metric tree. Instead of controlling the image of a single curve $t \mapsto T_{t}$ as in the previous lemma, we now have to control a family of curves $t \mapsto T_{t}(m)$, indexed by $m$. We review the arguments in the proof of Lemma 6.5, checking that the parameter $m$ introduces only finitely many new possibilities.

In order to make $f_{T}$ simplicial, we only have to subdivide $T_{0}(T)$. Because of the way $f_{T}$ was constructed (using the basepoint $P$ and its projections $P_{j}$ ), the subdivision of $T_{0}(T)$, and $f_{T}$ as a simplicial map, are independent of $m$. In particular, equivalence classes of couples $(\alpha, \beta)$ are defined independently of $m$. The subsegments $I_{c}(t)$ now depend on $m$, so we denote them by $I_{c}(t, m)$. Similarity is defined as in the proof 
of Lemma 6.5, but for couples: $(t, m)$ is similar to $\left(t^{\prime}, m^{\prime}\right)$ if there exist orientationpreserving homeomorphisms of $\varepsilon_{i}$ mapping each $I_{c}(t, m)$ to $I_{c}\left(t^{\prime}, m^{\prime}\right)$ (the length of $\varepsilon_{i}$ depends on $m$, but this is irrelevant since we only consider the combinatorial structure). There are only finitely many similarity classes, and we have seen in the previous proof that, as a non-metric tree, $T_{t}(m)$ depends only on the similarity class of $(t, m)$. This completes the proof of Lemma 6.4, hence also of the first assertion of Theorem 6.1.

Forester's deformation theorem. Before showing contractibility in the Gromov topology, we use Lemma 6.5 to give a proof of Forester's deformation theorem [16], which we state as follows:

Theorem 6.6. Let $T_{0}, T$ be simplicial (non-metric) trees with the same elliptic subgroups.

(1) There exist trees $T_{0}, T_{1}, \ldots, T_{k}=T$ such that $T_{i+1}$ is obtained from $T_{i}$ by an elementary expansion or an elementary collapse.

(2) If all edge stabilizers of $T_{0}$ and $T$ belong to a family $\mathcal{A}$ which is stable under taking subgroups, one may choose $T_{i}$ with edge stabilizers in $\mathcal{A}$.

The second assertion is not in [16], but it can be deduced from Forester's proof.

Proof. Using elementary collapses, we assume that $T_{0}$ is reduced. Since elliptic subgroups of $T_{0}$ are elliptic in $T$, we can map the vertex set of $T_{0}$ equivariantly to the vertex set of $T$. Adjacent vertices have distinct images by Remark 3.7, and we may choose metrics on $T_{0}$ and $T$ so that there exists a morphism $f: T_{0} \rightarrow T$.

We apply Lemma 6.5 (note that in its proof we only used the fact that $T_{0}$ and $T$ have the same elliptic subgroups). The set of intermediate trees $T_{t}$ is contained in a finite union of cones. By Proposition 5.2, $T_{t}$ is a continuous path in the weak topology. In particular, the union of all cones which meet this path is connected in that topology. Thus, there is a finite sequence of closed cones $T_{0} \in \mathcal{C}_{0}, \ldots, \mathcal{C}_{n} \ni T$ such that for all $i \in\{1, \ldots, n\}$, either $\mathcal{C}_{i}$ is a face of $\mathcal{C}_{i-1}$ or $\boldsymbol{C}_{i-1}$ is a face of $\mathcal{C}_{i}$. Now if $\mathcal{C}^{\prime}$ is a face of $\mathcal{C}$, trees of $\mathcal{C}^{\prime}$ are obtained from trees of $\mathcal{C}$ by an elementary collapse. This proves assertion (1).

Making $T_{0}$ reduced does not create new edge stabilizers. Edge stabilizers of $T_{t}$ are in $\mathcal{A}$ because there is a morphism $\psi_{t}: T_{t} \rightarrow T$. This proves assertion (2).

Contractibility in the Gromov topology. We now prove assertion (2) of Theorem 6.1. We assume that $T_{0}$ has finitely generated vertex stabilizers and we check that the condition in Lemma 6.2 holds. We first wish to say that the basepoint $P \in T$ depends continuously on $T$. As $P$ does not belong to a fixed space, we express it in the following way: 
Lemma 6.7. For any $a \in G$, the map $T \mapsto d(P$, a $P)$, from $\mathcal{D}$ to $\mathbb{R}$, is continuous.

Of course, $\mathcal{D}$ is now equipped with the Gromov topology.

Proof. We first consider the case when $\mathcal{D}$ is irreducible. We recall the definition of $P$. We fix $g, h \in G$ with $g, h,[g, h]$ hyperbolic in $\mathscr{D}$. If the axes $A_{g}$ and $A_{h}$ are disjoint, $P$ is the point of $A_{g}$ closest to $A_{h}$. If $A_{g}$ and $A_{h}$ meet, $P$ is the largest element of the segment $A_{g} \cap A_{h}$, with $A_{g}$ ordered so that $g$ acts as a positive translation.

Using the general formula $d(x, g x)=\ell(g)+2 d\left(x, A_{g}\right)$, it is easily checked that $P$ is characterized by the system

$$
\begin{aligned}
d(P, g P) & =\ell(g), \\
d(P, h P) & =\ell(h)+2 D, \\
d(g P, h P) & =\ell(g)+\ell(h)+2 D, \\
d\left(g P, h^{-1} P\right) & =\ell(g)+\ell(h)+2 D,
\end{aligned}
$$

with $D$ equal to the distance between $A_{g}$ and $A_{h}$ (see Figure 2).
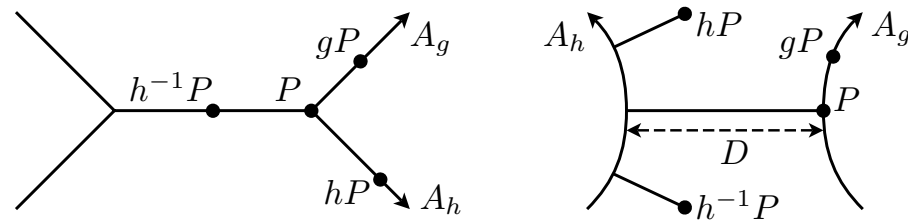

Figure 2. The basepoint $P$.

As in the proof of Proposition 5.7, this system is stable: if $\widetilde{P}$ satisfies each equation up to $\varepsilon$, then it is $C \varepsilon$-close to $P$, for some fixed number $C$. Furthermore, the righthand sides depend continuously on $T$ (see [28], Proposition 3.5, for the continuity of $D)$.

We can now prove that $d(P, a P)$ is continuous at any given $T$. Fix $\varepsilon>0$. Consider another $T^{\prime} \in \mathcal{D}$ and the corresponding system $\left(1^{\prime}\right)$. If $T^{\prime}$ is close enough to $T$ in the Gromov topology, then there exists a lift $\widetilde{P} \in T^{\prime}$ satisfying $\left(1^{\prime}\right)$ up to $\varepsilon$. We also require that $d(\widetilde{P}, a \widetilde{P})$ be $\varepsilon$-close to $d(P, a P)$. Then $\widetilde{P}$ is $C \varepsilon$-close to the basepoint $P^{\prime}$ of $T^{\prime}$, so $d\left(P^{\prime}, a P^{\prime}\right)$ is $(2 C+1) \varepsilon$-close to $d(P, a P)$.

When $\mathscr{D}$ is abelian, we fix two positive hyperbolic elements $g, h \in G$ with distinct axes, and $P$ is the endpoint of the ray $A_{g} \cup A_{h}$. The argument is the same as in the irreducible case, using the system $\left(1_{g h}\right)$ introduced in the proof of Proposition 5.7. 
More generally:

Lemma 6.8. Let $H_{1}, H_{2}$ be (possibly equal, possibly trivial) finitely generated subgroups of $G$ which are elliptic in D. Given $T \in \mathscr{D}$, let $P_{i} \in T$ be the projection of the basepoint $P$ onto the fixed point set of $H_{i}$. For any $a \in G$, the map $T \mapsto d\left(P_{1}, a P_{2}\right)$ is continuous.

Proof. We show continuity at a given $T \in \mathscr{D}$. For $i \in\{1,2\}$ fix a finite generating set $S_{i}$ for $H_{i}$. Choose $s_{i}$ in $S_{i}$ so that $d\left(P, s_{i} P\right)$ is maximal ( $s_{i}$ is not necessarily unique). The point $P_{i}$ is the midpoint of $\left[P, s_{i} P\right]$.

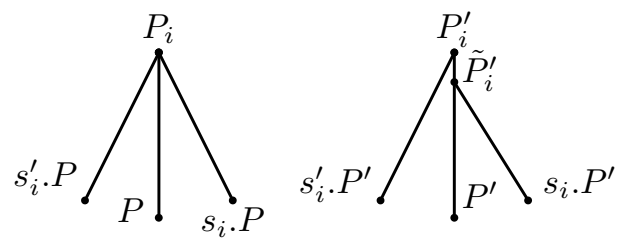

Figure 3. Proof of Lemma 6.8.

Now consider $T^{\prime}$ close to $T$ in $\mathscr{D}$. Define $P^{\prime}, P_{i}^{\prime}, s_{i}^{\prime}$ as in $T$ (see Figure 3). Note that $s_{i}$ is fixed, but $s_{i}^{\prime}$ may vary as $T^{\prime}$ varies. Let $\widetilde{P}_{i}^{\prime}$ be the midpoint of $\left[P^{\prime}, s_{i} P^{\prime}\right]$. Since $P_{i}^{\prime}$ is fixed by $s_{i}$, the point $\widetilde{P}_{i}^{\prime}$ is located on $\left[P^{\prime}, P_{i}^{\prime}\right]$ and $d\left(\widetilde{P}_{i}^{\prime}, P_{i}^{\prime}\right)=\frac{1}{2}\left(d\left(P^{\prime}, s_{i}^{\prime} P^{\prime}\right)-\right.$ $\left.d\left(P^{\prime}, s_{i} P^{\prime}\right)\right)$. Our choice of $s_{i}$ guarantees that $d(P, s P)-d\left(P, s_{i} P\right) \leq 0$ for every $s \in S_{i}$. Lemma 6.7 therefore implies that $d\left(\widetilde{P}_{i}^{\prime}, P_{i}^{\prime}\right)$ goes to 0 as $T^{\prime} \rightarrow T$.

Since distances between the four points $P, s_{1} P, a P, a s_{2} P$ vary continuously by Lemma 6.7 , the distance $d\left(\widetilde{P}_{1}^{\prime}, a \widetilde{P}_{2}^{\prime}\right)$ between the midpoints of $\left[P^{\prime}, s_{1} P^{\prime}\right]$ and $\left[a P^{\prime}, a s_{2} P^{\prime}\right]$ converges to $d\left(P_{1}, a P_{2}\right)$ as $T^{\prime} \rightarrow T$. Thus $d\left(P_{1}^{\prime}, a P_{2}^{\prime}\right) \rightarrow d\left(P_{1}, a P_{2}\right)$.

Since $f_{T}$ was defined using projections onto fixed point sets of vertex stabilizers of $T_{0}$, this lemma immediately implies that the condition in Lemma 6.2 holds if $T_{0}$ has finitely generated vertex groups. This completes the proof of Assertion (2) of Theorem 6.1.

The closure of an irreducible space is contractible. We now wish to extend the contraction to the closure $\bar{D}$ of an irreducible $\mathscr{D}$ (in the Gromov topology). Fix $g, h$ with $g, h,[g, h]$ hyperbolic. See Proposition 5.6 for properties of $\mathbb{R}$-trees $T \in \bar{D}$. In particular, $T$ is irreducible. The characteristic sets $A_{g}, A_{h}$ (axis or fixed point set) contain no tripod, and their intersection has length $\leq \ell(g)+\ell(h)$.

We have to change the definition of $P$, as there is no natural way to orient $A_{g}$ if $g$ is elliptic. We use the "symmetric" definition: $P$ is the midpoint of the segment 
$A_{g} \cap A_{h}$ if $A_{g}$ and $A_{h}$ meet, the midpoint of the "bridge" between them if they are disjoint.
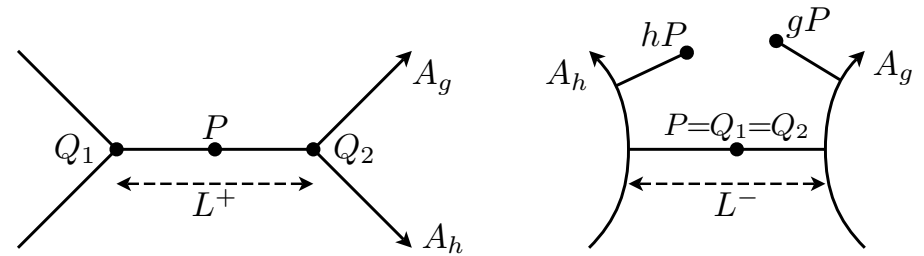

Figure 4. The "symmetric" basepoint.

The point $P$ is characterized by the following property (see Figure 4): there exist $Q_{1}, Q_{2}$ such that

$$
\begin{gathered}
d(P, g P)=d\left(Q_{1}, g Q_{1}\right)=d\left(Q_{2}, g Q_{2}\right)=\ell(g)+L^{-}, \\
d(P, h P)=d\left(Q_{1}, h Q_{1}\right)=d\left(Q_{2}, h Q_{2}\right)=\ell(h)+L^{-}, \\
d\left(P, Q_{1}\right)=d\left(P, Q_{2}\right)=L^{+} / 2, \\
d\left(Q_{1}, Q_{2}\right)=L^{+},
\end{gathered}
$$

where $L^{+}=\max (L, 0)$ and $L^{-}=\max (-L, 0)$, with $L \in \mathbb{R}$ equal to the length of $A_{g} \cap A_{h}$ if $A_{g} \cap A_{h}$ is nonempty, and equal to minus the distance between $A_{g}$ and $A_{h}$ if they are disjoint.

To see this, first suppose that $A_{g} \cap A_{h}=\emptyset$ (so $L^{+}=0$ ). The equations reduce to $d(P, g P)=\ell(g)+L^{-}$and $d(P, h P)=\ell(h)+L^{-}$(with $Q_{1}=Q_{2}=P$ ). They mean that $P$ has distance $L^{-} / 2$ to both $A_{g}$ and $A_{h}$. This determines it uniquely. If $A_{g} \cap A_{h} \neq \emptyset$, the first two equations mean that $P, Q_{1}, Q_{2}$ belong to $A_{g} \cap A_{h}$, the other two that the segment $\left[Q_{1}, Q_{2}\right]$ has length $L^{+}$and midpoint $P$. This uniquely determines $P$.

Furthermore, the system (2) is stable in the sense used above: if $\left(\widetilde{P}, \widetilde{Q}_{1}, \widetilde{Q}_{2}\right)$ is an approximate solution, then $\widetilde{P}$ is close to $P$.

To extend Lemmas 6.7 and 6.8 to $\bar{D}$, we have to know that $L$ is continuous on $\bar{D}$. It is continuous on $\mathscr{D}$ by [28], Proposition 3.5, but the argument of [28] breaks down when both $g$ and $h$ are elliptic in $T \in \bar{D}$. If this happens, first suppose that the fixed point sets of $g$ and $h$ are disjoint. Then we have $L=-\frac{1}{2}(\ell(g h)-\ell(g)-\ell(h))$ in $T$ and in nearby trees, so $L$ is continuous at $T$. If $g$ and $h$ have a common fixed point, we know that it is unique, so $L=0$ at $T$. Continuity at $T$ follows from the general inequality $|L| \leq \max \left(\ell(g)+\ell(h), \frac{1}{2} \ell(g h)\right)$.

The rest of the argument is now as before. The space $\bar{D}$ is contracted into a closed cone, as certain edges of $T_{0}(T)$ may have length 0 if $T \in \bar{D} \backslash \mathscr{D}$. 


\section{Non-ascending deformation spaces}

Given a $G$-tree $T$, we now view the quotient graph $\Gamma=T / G$ as a graph of groups. We often view a deformation space as a collection of marked graphs of groups (a marking of $\Gamma$ is an isomorphism from $G$ to the fundamental group of $\Gamma$, well defined up to composition with inner automorphisms). We say that $\Gamma$ is reduced if $T$ is.

We use the same notation (such as $v$ ) to denote a vertex of $T$ or of $\Gamma$. The corresponding group (vertex stabilizer or vertex group) is then denoted by $G_{v}$. Edges are denoted by letters such as $e$, with group $G_{e}$. An edge of $\Gamma$ is a loop if its endpoints are equal, a segment otherwise.

Slide moves. Let $e=v w$ and $f=v x$ be adjacent edges in a $G$-tree $T$, with $G_{f} \subset G_{e}$. Assume that $e, f$ are not in the same orbit as non-oriented edges. We define a new tree $T^{\prime}$ with the same vertex set as $T$ by replacing $f$ by an edge $f^{\prime}=w x$ and extending this operation equivariantly to the orbit of $f$. We say that $f$ slides across $e$ (see [18]). More generally, one can slide several edges $f_{i}$ satisfying $G_{f_{i}} \subset G_{e}$. Though sliding makes sense for metric trees, we shall apply it only in the context of non-metric trees.

The trees $T$ and $T^{\prime}$ belong to the same deformation space (they have the same vertex stabilizers). In fact, a slide move may be viewed as an elementary expansion followed by an elementary collapse (in the middle tree, $f$ is attached to the midpoint of $e$ ). The trees $T$ and $T^{\prime}$ even have the same edge stabilizers: since $G_{v} \cap G_{x} \subset$ $G_{v} \cap G_{w}$ and $G_{x} \cap G_{w} \subset G_{v}$, we have $G_{f^{\prime}}=G_{w} \cap G_{x}=G_{v} \cap G_{x}=G_{f}$.

It is easier to visualize a slide move on $\Gamma$, as one edge sliding across another (one or both may be loops). The number of vertices and edges of $\Gamma$ does not change. We usually describe a slide move by its action on $\Gamma$. There may exist different moves in $T$ with the same projection in $\Gamma$, but this will not be an issue here (compare [9]).

Non-ascending spaces: definition and examples. Given an edge $e=v w$ of $\Gamma$ (possibly with $v=w$ ), there are injections $G_{e} \hookrightarrow G_{v}$ and $G_{e} \hookrightarrow G_{w}$. We attach two labels to the edge $e$, one near $v$ and one near $w$. Each of these labels is $=$ or $\neq$, depending on whether the corresponding injection is onto or not.

With these notations, $\Gamma$ is reduced if and only if all labels $=$ are carried by loops. If a vertex $v$ has degree 2, at least one of the two labels near $v$ is $\neq$ (because there is no redundant vertex in $T$ ). If $e$ carries a label $=$ near $v$, then all other edges attached to $v$ may slide across $e$.

When viewed in $\Gamma$, an elementary collapse (see Section 3 ) is the collapse of a segment $e$ carrying at least one $=$ label; such an $e$ is called collapsible. The graph $\Gamma$ is reduced if and only if it contains no collapsible edge. All collapses considered here will be elementary collapses, so we often drop the word elementary.

We usually identify the edges of the collapsed graph with the edges of $\Gamma$ other than $e$, and a vertex of $\Gamma$ with its image in the collapsed graph. When $e$ is collapsed, a 
collapsible edge $f \neq e$ may become non-collapsible; a non-collapsible edge remains non-collapsible.

A strict ascending loop [18] is a loop $e$ of $\Gamma$ carrying exactly one $=$ and one $\neq$. The fundamental group of $e$, viewed as a graph of groups, is then a strictly ascending $\mathrm{HNN}$ extension. All edges adjacent to the basepoint of $e$ may slide around $e$.

A deformation space $\mathscr{D}$ is non-ascending if it is irreducible, and no $\Gamma$ in $\mathscr{D}$ contains a strict ascending loop. Note that genuine abelian spaces always contain graphs with a strict ascending loop.

The following observation will be very useful. Define an ascending circle as an embedded circle $C \subset \Gamma$ which may be oriented in such a way that all edges of $C$ carry $\mathrm{a}=$ label near their origin. If $\Gamma$ belongs to a non-ascending space, then edges of $C$ also carry a $=$ near their terminal point: otherwise, collapsing all edges of $C$ except one would create a strict ascending loop.

Proposition 7.1. Let $\mathfrak{D}$ be an irreducible deformation space. If one of the following conditions is satisfied, then $\mathscr{D}$ is non-ascending:

(1) No reduced graph of groups in $\mathfrak{D}$ contains a strict ascending loop.

(2) There exists a tree $T \in \mathscr{D}$ such that no fixed point set $\operatorname{Fix}(g)$, for $g \neq 1$, contains an infinite ray.

(3) No group in $\mathcal{A}_{\min }$ properly contains a conjugate of itself.

(4) There exist $T_{c} \in \mathcal{D}$ such that, for any $T \in \mathcal{D}$, there exists $\widetilde{T} \in \mathcal{D}$ such that both $T_{c}$ and $T$ may be obtained from $\widetilde{T}$ by collapses.

Recall that the weak acylindricity condition (2) holds for all trees in $\mathscr{D}$ if it holds for one (see Section 4 ), and that $\mathcal{A}_{\min }$ consists of groups fixing an edge in every $T \in \mathscr{D}$ (see Definition 4.8). Condition (3) holds if $\mathscr{D}$ is the canonical deformation space of a GBS group which does not contain any $\mathrm{BS}(1, n)$ with $n \geq 2$ (see [25]). Condition (4) means that $T_{c}$ is "compatible" with every $T \in \mathscr{D}$ : the graphs of groups $\Gamma_{c}$ and $\Gamma$ have a common refinement $\widetilde{\Gamma}$ in $\mathscr{D}$.

Proof. Starting with $\Gamma$ having a strict ascending loop $e$ based at $v$, we make it reduced without destroying the loop. First we collapse all collapsible edges which do not contain $v$, or have a $\neq$ label near $v$, or have two $=$ labels. This does not change $G_{v}$, so $e$ remains strictly ascending. If there remains a collapsible edge $f$, we change its $=$ label near $v$ to $\neq$ by sliding it around $e$. This makes $f$ non-collapsible. Iterate until all edges have been made non-collapsible.

If $\Gamma$ has a strict ascending loop $e$, the associated tree $T$ does not satisfy (2), and $G_{e}$ properly contains a conjugate $\left(G_{e} \in \mathcal{A}_{\min }\right.$ if $\Gamma$ is reduced).

It remains to consider (4). Recall that length functions of trees in $\mathscr{D}$ are biLipschitz equivalent (Theorem 3.8). To estimate the bi-Lipschitz constant, we restrict to trees all of whose edges have length 1 . Suppose that $T^{\prime}$ is obtained from $T$ by 
collapsing all edges in the orbit of an edge $e$. As pointed out in [16], components of this orbit have diameter at most 2 . This implies $\ell_{T} / 3 \leq \ell_{T^{\prime}} \leq \ell_{T}$ (at most two thirds of the edges in a given axis may be collapsed). If we can pass from $T$ to $T^{\prime}$ by $p$ elementary operations (expansions or collapses), we get $3^{-p} \ell_{T} \leq \ell_{T^{\prime}} \leq 3^{p} \ell_{T}$.

In the situation of (4), the number of operations needed to pass from $T_{c}$ to $T$ is bounded by $\left|\Gamma_{c}\right|+|\Gamma|$, where $|\Gamma|$ denotes the number of edges of the quotient graph, as we may assume that no edge of $\widetilde{T}$ gets collapsed in both $T_{c}$ and $T$.

A consequence of (4) is therefore the following: Given $k$, there exists $C$ such that, if $T, T^{\prime} \in \mathscr{D}$ have edges of length 1 and the quotient graphs $\Gamma, \Gamma^{\prime}$ have at most $k$ edges, then $\frac{1}{C} \ell_{T} \leq \ell_{T^{\prime}} \leq C \ell_{T}$. We now suppose that some reduced $\Gamma \in \mathscr{D}$ has a strict ascending loop $e$ based at a vertex $v$, and we obtain a contradiction to this assertion.

Let $f$ be another edge adjacent to $v$ (it exists because $\mathscr{D}$ is irreducible). We may assume that it carries no $=$ label (if it does, then it is a loop at $v$ because $\Gamma$ is reduced, and we get rid of $=$ labels by sliding around $e$ ).

Define a graph $\Gamma_{n}$, with Bass-Serre tree $T_{n}$, by sliding $f n$ times around $e$ (oriented from its $=$ label to its $\neq$ label). Recall that all trees $T_{n}$ have the same vertex set. Consider a lift $v_{0} x$ of $f$ to $T_{0}=T$, with $v_{0}$ lifting $v$. It is slid to a position $v_{n} x$ in $T_{n}$. For $0 \leq i \leq n$, the distance between $v_{n}$ and $x$ in $T_{i}$ is $n-i+1$ (the geodesic is $\left.v_{n} v_{n-1} \ldots v_{i} x\right)$.

Choose $g \in G$ fixing $x$ but not $v_{0}$ (it exists because $f$ carries no $=$ ), and $h_{n}$ fixing $v_{n}$ but not $v_{n-1}$ (the sequence $G_{v_{n}}$ is strictly ascending because $e$ is). The distance between the fixed point sets of $g$ and $h_{n}$ in $T_{i}$ is $d_{T_{i}}\left(v_{n}, x\right)=n-i+1$, so $\ell_{T_{i}}\left(g h_{n}\right)=2(n-i+1)$. Writing this for $i=0$ and $i=n$, we see that the bi-Lipschitz constant between $\ell_{T}$ and $\ell_{T_{n}}$ goes to infinity with $n$, contradicting the consequence of (4) stated above.

Connecting trees by slide moves. In the rest of this section we only consider nonascending spaces.

It is easy to show that, in a non-ascending deformation space, every elliptic subgroup is contained in a maximal one. If $T$ is reduced, there is a bijection between the set of vertices of $\Gamma$ and the set of conjugacy classes of maximal elliptic subgroups (this is the vertical set $\mathcal{M}$ of Section 4). The next result will provide more precise information. We consider trees as simplicial, non-metric, objects (or we restrict to trees with edges of length 1).

Theorem 7.2. Let $\mathfrak{D}$ be a non-ascending deformation space. Any two reduced simplicial trees $T, T^{\prime} \in \mathbb{D}$ may be connected by a finite sequence of slides.

Conversely, performing a slide move on a reduced tree in a non-ascending space yields a reduced tree. 
This theorem was proved by Forester [18] for the canonical deformation space of GBS groups. His proof works in the general situation. We give a different argument.

Proof. By Remark 3.6, we may assume that $T, T^{\prime}$ are different reductions of a tree $S$. We also assume that no edge of $S$ gets collapsed in both $T$ and $T^{\prime}$. We work with the quotient graphs of groups $\Gamma, \Gamma^{\prime}, \Theta$ of $T, T^{\prime}, S$. We first show that there exist edges $e, e^{\prime}$ of $\Theta$ with a common vertex $v$ such that both $e$ and $e^{\prime}$ carry a = near $v, e$ gets collapsed in $\Gamma^{\prime}$, and $e^{\prime}$ gets collapsed in $\Gamma$.

Since $\Theta$ is not reduced, we can find an edge $e_{1}$ of $\Theta$ which gets collapsed in $\Gamma^{\prime}$. Let $v_{1}$ be an endpoint of $e_{1}$ with label $=$. Since $e_{1}$ is not collapsed in $\Gamma$, some edge $e_{1}^{\prime}$ of $\Theta$ with endpoints $v_{1}$, $v_{1}^{\prime}$ gets collapsed in $\Gamma$. If the label of $e_{1}^{\prime}$ at $v_{1}^{\prime}$ is $\neq$, its label at $v_{1}$ is $=$ and we are done. If not, we repeat the argument, using $e_{1}^{\prime}, v_{1}^{\prime}$ instead of $e_{1}, v_{1}$. We obtain edges $e_{2}, e_{2}^{\prime}, e_{3}, \ldots$ which get collapsed in $\Gamma$ or $\Gamma^{\prime}$ alternatively. If the process does not stop, we eventually create an ascending circle $C$. Since $\mathscr{D}$ is non-ascending, $C$ carries only $=$ labels and we can find $e, e^{\prime}$.

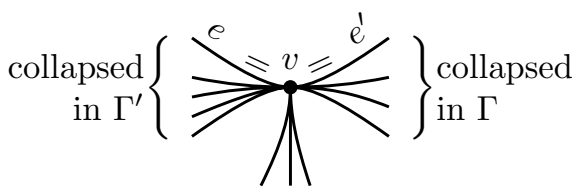

$\Theta$

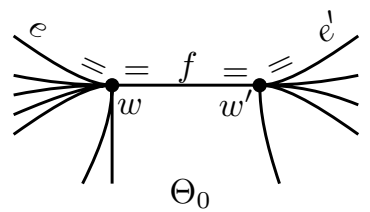

$\Theta_{0}$

Figure 5. Proof of Theorem 7.2.

Let $e, e^{\prime}$ be as constructed. We define a new graph of groups $\Theta_{0}$ by performing an elementary expansion at $v$ in $\Theta$ as follows (see Figure 5). We replace $v$ by a new edge $f=w w^{\prime}$ carrying two $=$ labels. Edges of $\Theta$ incident to $v$ are attached to $w$ or $w^{\prime}$, the only restriction being that edges collapsed in $\Gamma^{\prime}$ (resp. $\Gamma$ ) are attached to $w$ (resp. $w^{\prime}$ ) (changing the attachment point of an edge amounts to sliding it across $f$ ). In particular, the edge $e\left(\right.$ resp. $\left.e^{\prime}\right)$ is attached to $w\left(\right.$ resp. $\left.w^{\prime}\right)$. It carries a label $=$ at $w$ (resp. $\left.w^{\prime}\right)$.

Let $F_{0}$ be the image in $\Theta_{0}$ of the subforest of $\Theta$ which gets collapsed in $\Gamma$ (it contains $e^{\prime}$ ). Collapsing $F_{0} \cup f$ in $\Theta_{0}$ yields $\Gamma$. Since no edge of $F_{0}$ is attached to $w$, we may also collapse $F_{0} \cup e$. This gives a graph $\Gamma_{1}$ which differs from $\Gamma$ by slide moves (the middle graph is obtained from $\Theta_{0}$ by collapsing $F_{0}$ ). Define $\Gamma_{1}^{\prime}$ similarly, using $\Gamma^{\prime}$ instead of $\Gamma$. The graphs $\Gamma_{1}$ and $\Gamma_{1}^{\prime}$ are both reductions of the graph $\Theta_{1}$ obtained from $\Theta_{0}$ by collapsing $e \cup e^{\prime}$. Since $\Theta_{1}$ has one less edge than $\Theta$, iterating this construction yields a sequence of slides connecting $\Gamma$ and $\Gamma^{\prime}$.

Corollary 7.3. If $\mathscr{D}$ is non-ascending, all reduced trees in $\mathscr{D}$ have the same vertex and edge stabilizers; given two reduced trees in $\mathscr{D}$, there are $G$-equivariant bijections $\psi_{V}, \psi_{E}$ between their vertex and edge sets. 
By [14], the existence of $\psi_{V}$ always implies that of $\psi_{E}$. In general, $\psi_{V}$ and $\psi_{E}$ are not compatible with the incidence relations.

A finite dimensional retract. Recall that we only consider graphs of groups in non-ascending deformation spaces. All collapses considered here are elementary.

As mentioned above, a collapsible edge of $\Gamma$ may become non-collapsible when other edges are collapsed. This motivates the following definition.

An edge $e$ of $\Gamma$ is a surviving edge if it is non-collapsible, or may be made noncollapsible by collapsing other edges. In other words, $e$ is a surviving edge if and only if one can make $\Gamma$ reduced without collapsing $e$.

Recall that there are two types of non-collapsible edges: segments carrying two $\neq$ labels, and loops. In order to describe surviving edges, we define two types of subgraphs $\gamma$ of $\Gamma$ in terms of their topology and the labels they carry:

- $\gamma=\left(e_{1}, \ldots, e_{k}\right)$ is an embedded edge-path (homeomorphic to [0,1]); all labels carried by $\gamma$ at interior vertices are $=$, but the labels carried by $e_{1}$ at its origin, and by $e_{k}$ at its terminal point, are $\neq$ (when $k=1$, this is just a non-collapsible segment).

- $\gamma$ is homeomorphic to a circle; all labels carried by $\gamma$ are $=$, except possibly those at a special vertex $v_{0}$ (if there is a special vertex, both labels at $v_{0}$ are $\neq$ by the non-ascending assumption).

We call such a $\gamma$ a shelter. Note that one-edge shelters are exactly non-collapsible edges. Any edge $e$ contained in a shelter $\gamma$ is a surviving edge, since it becomes non-collapsible when the other edges of $\gamma$ are collapsed. The following lemma will imply the converse (every surviving edge is contained in a shelter).

Lemma 7.4. Let $\Gamma$ be a graph of groups belonging to a non-ascending deformation space, and let $\Gamma^{\prime}$ be obtained from $\Gamma$ by collapsing a collapsible edge $f$. An edge $e \neq f$ is contained in a shelter of $\Gamma$ if and only if its image $e^{\prime}$ in $\Gamma^{\prime}$ is contained in a shelter.

Proof. This is just a statement about graphs with $\mathrm{a}=, \neq$ labeling. The proof is not difficult, but there will be several cases to consider.

Let $v, w$ be the vertices of $f$. They are distinct and $f$ carries at least one $=$ label, say near $v$. When $f$ is collapsed, labels carried by other edges near $w$ do not change (the vertex group does not change). $\mathrm{A} \neq$ label near $v$ remains $\neq . \mathrm{A}=$ label gets replaced by the label of $f$ near $w$.

Assume that $e$ is contained in a shelter $\gamma$. If $f$ is disjoint from $\gamma$, or contained in $\gamma$, or if $f \cap \gamma=\{w\}$, the image of $\gamma$ in $\Gamma^{\prime}$ is a shelter. If $f \cap \gamma=\{v\}$, the image of $\gamma$ consists of one or two shelters. Now assume $f \cap \gamma=\{v, w\}$. If the label of $f$ near $w$ is $\neq$, the non-ascending hypothesis implies that $w$ must be an endpoint of $\gamma$ or the special vertex $v_{0}$. The image of $\gamma$ in $\Gamma^{\prime}$ is homeomorphic to a circle, or to a wedge of two circles, or to the union of a circle and an interval. One checks that each of these circles or intervals is a shelter. We have proved one direction of the lemma. 
Conversely, assume that $e^{\prime}$ is contained in a shelter $\gamma^{\prime}$. Let $w^{\prime}$ be the image of $v$ and $w$ in $\Gamma^{\prime}$ (so $\Gamma$ is obtained from $\Gamma^{\prime}$ by expanding $w^{\prime}$ into $f$ ). We may assume that $w^{\prime} \in \gamma^{\prime}$. Let $\gamma_{0}$ be the subgraph of $\Gamma$ consisting of edges projecting onto the edges of $\gamma^{\prime}$ (it has one or two components). Edges of $\gamma_{0}$ near $f$ may be attached to either $v$ or $w$. We may assume that at least one is attached to $v$ (otherwise, $\gamma_{0}$ is obviously a shelter).

First assume that the label of $f$ near $w$ is $=$. The only non-trivial case is when $\gamma_{0}$ has one edge attached to $v$ and one to $w$. If $w^{\prime}$ is the special vertex of a circular $\gamma^{\prime}$, then $\gamma_{0}$ is a shelter (homeomorphic to an interval). If not, then $\gamma_{0} \cup f$ is a shelter.

Now suppose that the label of $f$ near $w$ is $\neq$. Then $w^{\prime}$ has to be an endpoint of $\gamma^{\prime}$, or its special vertex $v_{0}$, since otherwise no edge of $\gamma_{0}$ could be attached to $v$. If it is an endpoint, then $\gamma_{0}$ is a shelter if the label of $\gamma_{0}$ near $v$ is $\neq$, and $\gamma_{0} \cup f$ is a shelter if the label is $=$. The same conclusion holds if $w^{\prime}$ is the special vertex of $\gamma^{\prime}$ and $\gamma_{0}$ has an edge attached to $w$. If $w^{\prime}=v_{0}$ and $\gamma_{0}$ has two edges attached to $v$, then $\gamma_{0}$ is a shelter.

Corollary 7.5. Let $\Gamma$ be a graph of groups belonging to a non-ascending deformation space.

(1) An edge is a surviving edge if and only if it is contained in a shelter.

(2) Let $\Gamma^{\prime}$ be obtained from $\Gamma$ by collapsing a collapsible edge $f$. An edge $e \neq f$ is a surviving edge of $\Gamma$ if and only if its image $e^{\prime}$ in $\Gamma^{\prime}$ is a surviving edge.

Proof. We have seen that edges contained in a shelter are surviving edges. If $e$ is a surviving edge, one may collapse edges other than $e$ to obtain a reduced $\Gamma_{r}$. The image of $e$ in $\Gamma_{r}$ is non-collapsible, so is a one-edge shelter. By the "if" part of the lemma, $e$ is contained in a shelter of $\Gamma$. Assertion (2) is just a rewording of the lemma.

Let $\mathscr{C} \subset \mathbb{D}$ be the set of graphs of groups all of whose edges are surviving edges. It is stable under collapses, so its projection $P \mathscr{G} \subset P \mathscr{D}$ is a subcomplex. Using shelters, it is easy to see that trees corresponding to graphs $\Gamma$ in $\mathcal{G}$ are BF-reduced (as defined in Section 3): if a vertex $v$ of $\Gamma$ has valence 2 and the labels near $v$ are $=, \neq$, the edge carrying the $=$ label cannot be contained in a shelter. Proposition 4.2 implies that $\mathcal{G}$ is finite dimensional.

Theorem 7.6. Let $\mathfrak{D}$ be a non-ascending deformation space, equipped with the weak topology. There is a deformation retraction $r$ from $P \mathscr{D}$ onto the finite dimensional subcomplex $P \mathscr{G}$. If $F \subset \operatorname{Out}(G)$ leaves $D$ invariant, then $r$ is $F$-equivariant.

Proof. The deformation retraction is simply given by letting the length of the nonsurviving edges go linearly to 0 . It maps a given closed simplex into itself in a continuous way by assertion (2) of Corollary 7.5, so it is continuous in the weak 
topology. The deformation is clearly equivariant with respect to the group of automorphisms leaving $\mathscr{D}$ invariant (its action on a graph of groups consists in changing the marking).

We have seen that all reduced trees in $\mathscr{D}$ have the same edge stabilizers (Corollary 7.3). The same property holds for trees in $\mathcal{E}$, since all edges of graphs in $\mathcal{E}$ are surviving edges. This shows that $\mathcal{E}$ is contained in the reduced deformation space $\mathscr{D}_{r}$ defined in Section 4.

Examples. In these examples, $G$ is a GBS group, $\mathscr{D}$ is its canonical deformation space (see the end of Section 3), and $\mathcal{E}$ is the set of graphs of groups all of whose edges are surviving edges.

- Let $G=\left\langle a, b, c, t \mid a^{2}=b^{6}, b^{2}=c^{2}, t c^{3} t^{-1}=b^{9}\right\rangle$. Using arguments from [18], it may be shown that $\mathscr{D}$ is non-ascending (although $G$ contains $\operatorname{BS}(1,3)$ ). The subcomplex $\mathcal{G}$ is 4-dimensional, but $\mathscr{D}_{r}$ is infinite dimensional.

- Let $G=\mathrm{BS}(2,4)$. In this case, $\mathcal{E}$ is not a subcomplex and there is no retraction from $\mathcal{D}$ to $\mathcal{G}$. Clay has shown, however, that there always is a retraction from $\mathscr{D}$ to the barycentric spine of $\mathcal{E}$ [9].

- Let $G=\left\langle a, s, t \mid s a s^{-1}=a^{2}, t a t^{-1}=a^{2}\right\rangle$. By sliding one loop many times around the other and performing elementary expansions, one constructs graphs of groups with an ascending circle of arbitrarily large length. It follows that $\mathcal{E}$ is infinite dimensional.

\section{Applications to automorphisms}

Restriction. Up to now, we have been working with a single finitely generated group $G$. In this subsection, we also consider a finitely generated group $\widehat{G}$ containing $G$ as a normal subgroup. This study will be used in the next subsection, with $G$ a centerless group and $\widehat{G}$ the preimage of some $F \subset \operatorname{Out}(G)$ under the natural map $\operatorname{Aut}(G) \rightarrow \operatorname{Out}(G)$.

The results of the present subsection also hold (and are easier to prove) if $G$ has finite index in $\widehat{G}$ (without being normal) and more generally if any $h \in \widehat{G}$ has a power in $G$.

We shall consider irreducible trees. Recall that they are determined by their length function $\ell$. The Gromov topology and the axes topology coincide on the set of irreducible trees (see Section 5). In this section it will be more convenient to use the axes topology (i.e. to work with length functions).

Any $\widehat{G}$-tree $T$ may be viewed as a $G$-tree. We call this a restriction. We sometimes write $T_{\widehat{G}}$ and $T_{G}$ instead of simply $T$ to emphasize which group we are considering. Note that $T_{\widehat{G}}$ is obviously minimal and irreducible if $T_{G}$ is. 
Lemma 8.1. Let $G \triangleleft \widehat{G}$ be a pair of finitely generated groups with $G$ normal in $\widehat{G}$. Let $T$ be an irreducible $\widehat{G}$-tree, with length function $\ell$. Assume that $G$ is not contained in the kernel of the action.

(1) The $G$-tree $T_{G}$ is minimal and irreducible.

(2) For any $h \in \widehat{G}$, one has

$$
\ell(h)=\sup _{g \in G} \limsup _{k \rightarrow+\infty} \frac{1}{2 k} \ell\left(\left[g, h^{k}\right]\right) .
$$

(3) $\ell$ is determined by its restriction to $G$.

The kernel of the action is the set of elements acting on $T$ as the identity.

Proof. If $G$ is elliptic, its fixed subtree is $\widehat{G}$-invariant because $G$ is normal, so equals $T$ by minimality of $T_{\widehat{G}}$; this means that $G$ is contained in the kernel. If there is a unique $G$-invariant end, or a unique $G$-invariant line, this end or line is $\widehat{G}$-invariant, contradicting irreducibility of $T_{\widehat{G}}$. The only remaining possibility is that $T_{G}$ is irreducible. It is minimal because the unique minimal $G$-invariant subtree is $\widehat{G}$-invariant and $T_{\widehat{G}}$ is minimal.

Given any $h$, denote by $A_{h}$ its axis or fixed point set. Recall the formula $d(x, h x)=$ $\ell(h)+2 d\left(x, A_{h}\right)$, valid for any $x \in T$, and the general inequality $\ell\left(h h^{\prime}\right) \leq \ell(h)+$ $\ell\left(h^{\prime}\right)+2 d\left(A_{h}, A_{h^{\prime}}\right)$, obtained by computing $d\left(h h^{\prime} x, x\right)$ for an $x \in A_{h^{\prime}}$ such that $h^{\prime} x$ minimizes the distance to $A_{h}$. Writing $\left[g, h^{k}\right]=g\left(h^{k} g^{-1} h^{-k}\right)$, we obtain

$$
\ell\left(\left[g, h^{k}\right]\right) \leq 2 \ell(g)+2 d\left(A_{g}, h^{k} A_{g}\right) \leq 2 \ell(g)+2 k \ell(h)+4 d\left(A_{g}, A_{h}\right) .
$$

This shows that $\ell(h)$ is bigger than the sup appearing in the statement of the lemma.

To prove the opposite inequality, we may assume that $h$ is hyperbolic. By assertion (1), there exists a hyperbolic $g \in G$ with $A_{g} \cap A_{h}$ compact. For $k$ large, $A_{g}$ and $A_{h^{k} g^{-1} h^{-k}}=h^{k} A_{g}$ are disjoint, and their distance is $k \ell(h)+o(k)$. Since $\ell\left(g g^{\prime}\right)=\ell(g)+\ell\left(g^{\prime}\right)+2 d\left(A_{g}, A_{g^{\prime}}\right)$ for hyperbolic elements with disjoint axes, we have $\ell\left(\left[g, h^{k}\right]\right)=2 k \ell(h)+o(k)$.

Assertion (3) follows from (2), since $\left[g, h^{k}\right] \in G$ if $g \in G$.

Let $G, \widehat{G}$ be as in the lemma. Consider any $\widehat{G}$-tree $T_{\widehat{G}}$ and its restriction $T_{G}$. Elliptic subgroups of $T_{G}$ are precisely intersections of elliptic subgroups of $T_{\widehat{G}}$ with $G$. Restriction therefore sends a given deformation space $\mathscr{D}_{\widehat{G}}$ of $\widehat{G}$-trees into a deformation space $\mathscr{D}_{G}$ of $G$-trees. We denote this map by $i: \mathscr{D}_{\widehat{G}} \rightarrow \mathscr{D}_{G}$. By Lemma 8.1, it is injective if $\mathscr{D}_{\widehat{G}}$ is irreducible and $G$ is not elliptic in $\mathscr{D}_{\widehat{G}}$.

Lemma 8.2. Let $G \triangleleft \widehat{G}$ be a pair of finitely generated groups with $G$ normal in $\widehat{G}$.

(1) An irreducible deformation space of $G$-trees contains the image of at most one deformation space of $\widehat{G}$-trees. 
(2) If $\mathscr{D}_{\widehat{G}}$ is an irreducible deformation space of $\widehat{G}$-trees in which $G$ is not elliptic, restriction induces a homeomorphism from $D_{\widehat{G}}$ onto a closed subspace of an irreducible deformation space $\mathscr{D}_{G}$ of $G$-trees.

Assertion (2) is true both in the Gromov (or axes) topology and in the weak topology.

Proof. Recall that two irreducible trees belong to the same deformation space if and only if their length functions are bi-Lipschitz equivalent (Theorem 3.8). If the restrictions of $\ell$ and $\ell^{\prime}$ to $G$ are bi-Lipschitz equivalent, then $\ell$ and $\ell^{\prime}$ are bi-Lipschitz equivalent by assertion (2) of Lemma 8.1. This shows the first assertion.

Under the hypotheses of (2), we have seen that restriction induces an injection $i: \mathscr{D}_{\widehat{G}} \rightarrow \mathscr{D}_{G}$ into an irreducible space. We now study the topological properties of $i$.

First consider the weak topology. Given a $\widehat{G}$-tree $T_{\widehat{G}}$, restriction maps the closed cone spanned by $T_{\widehat{G}}$ linearly into the closed cone of $\mathscr{D}_{G}$ spanned by $T_{G}$ (as in Section 5, we parametrize cones by edge-lengths). The image consists of all trees satisfying certain equalities between edge-lengths. In particular, at most one open cone of $D_{\widehat{G}}$ maps into a given open cone of $D_{G}$. Assertion (2) for the weak topology follows from these observations.

In the axes topology, the only obvious fact is continuity of $i$. To prove that $i$ is a homeomorphism onto a closed set, we suppose that $\ell_{n}$ is a sequence of length functions on $\widehat{G}$, associated to trees in $\mathscr{D}_{\widehat{G}}$, whose restrictions to $G$ converge to a nontrivial length function $\ell_{G}$ associated to a tree $T_{G}$ in $\mathcal{D}_{G}$. We show that $\ell_{n}$ converges to a length function associated to a tree in $\mathscr{D}_{\widehat{G}}$.

Since the set of projectivized length functions on $\widehat{G}$ is compact [11], we can find numbers $a_{n}>0$ such that $a_{n} \ell_{n}$ converges to a non-trivial length function $\ell$ on $\widehat{G}$ (after replacing $\ell_{n}$ by a subsequence). The sequence $a_{n}$ is bounded because $\ell_{G}$ is non-trivial. We have to bound it away from 0 .

Let $T$ be the $\mathbb{R}$-tree with length function $\ell$. It is irreducible by Proposition 5.6. If $g \in G$ is hyperbolic in $\mathscr{D}_{\widehat{G}}$, it may be elliptic in $T$, but cannot fix a tripod (see Proposition 5.6). This implies that $G$ is not contained in the kernel of the action. In particular (see Lemma 8.1), there exists $g_{0} \in G$ with $\ell\left(g_{0}\right) \neq 0$. Since $\ell_{n}\left(g_{0}\right)$ converges to $\ell_{G}\left(g_{0}\right)$ and $a_{n} \ell_{n}\left(g_{0}\right)$ converges to a non-zero number, the bounded sequence $a_{n}$ has a positive limit, which we may assume to be 1 . This implies that $\ell_{n}$ converges to $\ell$, and $\ell_{G}$ is the restriction of $\ell$ to $G$. In particular, $T_{G}$ is the restriction of $T$, and $T$ is simplicial. It is in $\mathscr{D}_{\widehat{G}}$ by assertion (1). This completes the proof of assertion (2) for the axes topology.

Fixed point sets. We return to the study of a single finitely generated group $G$. There is a natural action of $\operatorname{Out}(G)$ on the set of $G$-trees, given by precomposition. If a subgroup of $\operatorname{Out}(G)$ fixes a tree, it acts on the deformation space containing it. 
Theorem 8.3. Let $D$ be an irreducible deformation space. If a finitely generated group $F \subset \operatorname{Out}(G)$ fixes a tree $T_{0} \in \mathscr{D}$, the fixed point set of $F$ in $D$ is contractible in the weak topology.

Proof. Let $\widehat{G}$ be the preimage of $F$ by the natural map $\operatorname{Aut}(G) \rightarrow \operatorname{Out}(G)$. First assume that $G$ has trivial center. It embeds into $\operatorname{Aut}(G)$, as inner automorphisms, and $\widehat{G}$ is a finitely generated group containing $G$ as a normal subgroup.

The key observation is that a $G$-tree $T_{G}$ is $F$-invariant if and only if the action of $G$ on $T$ extends to an action of $\widehat{G}$. The fixed point set of $F$ in $\mathscr{D}$ is homeomorphic to an irreducible deformation space $D_{\hat{G}}$ by Lemma 8.2, so is contractible by Theorem 6.1.

If $G$ has a non-trivial center, $\widehat{G}$ contains $G / Z(G)$ as a normal subgroup and we simply view $\mathscr{D}$ as a deformation space of $G / Z(G)$-trees (see the last example in Section 3).

Theorem 8.3 also holds in the Gromov topology, under the extra assumption that $T_{0}$ has finitely generated vertex stabilizers (as a $G$-tree, hence also as a $\widehat{G}$-tree).

Corollary 8.4. Let $\mathfrak{D}$ be an irreducible deformation space. Suppose that $\mathscr{D}$ is finite dimensional, or non-ascending. If a solvable finite group $F \subset \operatorname{Out}(G)$ leaves $D$ invariant, then $F$ has a fixed point in $D$.

Proof. By induction on the order of $F$. If $F$ is not trivial, it has a normal subgroup $F_{1}$ with $F / F_{1}$ cyclic of prime order $p$. By induction, $F_{1}$ fixes a point in $\mathscr{D}$. Let $\mathscr{D}_{1} \subset \mathcal{D}$ be its fixed point set.

First assume that $\mathscr{D}$ is finite dimensional. Then $\mathscr{D}_{1}$ is finite dimensional, and contractible by Theorem 8.3. The group $F / F_{1} \simeq \mathbb{Z} / p \mathbb{Z}$ acts on $\mathscr{D}_{1}$ and has a fixed point (otherwise, it would have a finite dimensional classifying space). This means that $F$ has a fixed point in $\mathscr{D}$.

If $\mathscr{D}$ is non-ascending, we consider the finite dimensional subcomplex $\mathscr{C} \subset \mathscr{D}$ constructed in Section 7. The deformation retraction $\mathscr{D} \rightarrow \mathscr{E}$ is $F$-equivariant. Let $D_{1}$ (resp. $\mathscr{E}_{1}$ ) be the fixed point set of $F_{1}$ in $\mathscr{D}$ (resp. $\mathscr{E}$ ). By induction, $\mathscr{D}_{1}$ is nonempty. As it is contractible, we deduce that $\mathscr{E}_{1}$ is contractible and we conclude by considering the action of $F / F_{1}$ on $\mathscr{E}_{1}$.

Locally finite trees. In this subsection, we consider a deformation space $\mathscr{D}$ consisting of locally finite trees and the projectivized space $P \mathscr{D}$. We let $\operatorname{Out}_{\mathscr{D}}(G)$ be the subgroup of $\operatorname{Out}(G)$ leaving $\mathscr{D}$ invariant.

We say that $H \subset G$ is a stabilizer if it is a vertex or edge stabilizer in some $T \in \mathscr{D}$. All stabilizers are commensurable as subgroups of $G$ : if $H_{1}, H_{2}$ are stabilizers, then $H_{1} \cap H_{2}$ has finite index in both $H_{1}$ and $H_{2}$ (this is clear if $H_{1}, H_{2}$ are stabilizers in the same tree, and also if they are stabilizers in trees differing by an elementary expansion). 
A group $H$ (possibly infinitely generated) is small [3] if there is no $H$-tree in which axes of two hyperbolic elements intersect in a compact set. Any group not containing $F_{2}$ is small. Smallness is not stable under taking subgroups, even of finite index.

To remedy this, we say that $H \subset G$ is small in $G$ if there is no $G$-tree in which axes of two hyperbolic elements of $H$ intersect in a compact set. Of course, any small group contained in $G$ is small in $G$, and being small in $G$ is stable under taking subgroups. It is also a commensurability invariant.

Lemma 8.5. Given $G$, there exists at most one irreducible deformation space consisting of locally finite trees whose stabilizers are small in $G$.

Proof. This is proved in [16] in the case of stabilizers $\mathbb{Z}$, in [8] for slender stabilizers (recall that a group is slender [15] if all of its subgroups are finitely generated). We use the same argument to determine elliptic subgroups algebraically: if $T$ is locally finite and stabilizers are small in $G$, then a subgroup $H \subset G$ is elliptic if and only if it is contained in a subgroup which is small in $G$ and commensurable to all its conjugates.

The "only if" direction is obvious. Conversely, suppose that $H$ is small in $G$, commensurable to all its conjugates, but not elliptic. It is easy to check that $H$ acts on $T$ with a unique fixed end or a unique invariant line. This line or end is $G$-invariant, contradicting irreducibility.

A basic invariant of $\mathcal{D}$ is the modular homomorphism $\Delta: G \rightarrow \mathbb{Q}^{+*}$ with values in the multiplicative group of positive rationals [2], [18]. It may be defined by choosing a stabilizer $H$ and setting $\Delta(g)=\frac{\left[H: H \cap g \mathrm{Hg}^{-1}\right]}{\left[g \mathrm{Hg}^{-1}: \mathrm{H \cap gH}^{-1}\right]}$. Given $T \in \mathscr{D}$, one has $\Delta=\tau \circ \theta$, where $\theta$ is the natural epimorphism from $G$ to the topological fundamental group of the quotient graph $\Gamma$ (see the beginning of Section 4 ), and $\tau$ is defined on a loop $\gamma=\left(e_{1}, \ldots, e_{k}\right)$ by $\tau(\gamma)=\prod_{j=1}^{k} \frac{i\left(e_{j}\right)}{i\left(\bar{e}_{j}\right)}$, denoting by $i\left(e_{j}\right)\left(\operatorname{resp} . i\left(\bar{e}_{j}\right)\right)$ the index of the edge group in the vertex group at the origin (resp. endpoint) of $e_{j}$.

Remark. There is a more refined invariant, with values in the abstract commensurator of $H$. For GBS groups, it is the (signed) modular homomorphism used in [18] or [25].

As in [18], we say that $\mathscr{D}$ has no non-trivial integer modulus if the image of $\Delta$ contains no integer $n>1$ (when vertex groups are isomorphic to $\mathbb{Z}$, this is equivalent to saying that $G$ does not contain a solvable Baumslag-Solitar group BS $(1, n)$ with $n>1$, see [25]). An irreducible deformation space with no non-trivial integer modulus is obviously non-ascending.

Proposition 8.6. Let $H$ be a finitely generated subgroup of $G$ such that $H$, and every group commensurable to $H$, has finite outer automorphism group. Let $D$ be 
an irreducible deformation space consisting of locally finite G-trees with stabilizers commensurable to $H$.

(1) If $H$ is small in $G$, then $\operatorname{Out}_{\mathscr{D}}(G)=\operatorname{Out}(G)$.

(2) If $H$, and all its finite index subgroups, have finite center, then $\operatorname{Out}_{\mathscr{D}}(G)$ acts on $D$ with finite stabilizers.

(3) If there is no non-trivial integer modulus, then $\operatorname{Out}_{\mathscr{D}}(G)$ acts on $P \mathscr{D}$ with finitely many orbits of simplices.

Proof. (1) follows from Lemma 8.5.

For (2), consider $T \in \mathcal{D}$. We study its stabilizer $\operatorname{Out}^{T}(G) \subset \operatorname{Out}(G)$ using results and notations from [24]. Since all edge and vertex stabilizers have Out finite, the group of twists $\mathcal{T}$ has finite index in $\operatorname{Out}^{T}(G)$ ([24], Theorem 1.6). This group $\mathcal{T}$ is a quotient of a finite direct product of centralizers $Z_{G_{v}}\left(G_{e}\right)$, with $G_{e}$ an edge stabilizer and $G_{v}$ an adjacent vertex stabilizer. The hypothesis of (2) implies that all groups commensurable with $H$ have finite center, so all these centralizers are finite.

(3) was proved by Forester for GBS groups [18]. We generalize his argument. Given $T \in \mathcal{D}$, consider the quotient graph of groups $\Gamma$. The hypothesis about moduli implies that there is a uniform bound $N$ (independent of $T$ ) for the index of an edge group in an adjacent vertex group ([18], Theorem 8.2).

If $H_{1}, H_{2}$ are commensurable to $H$, we note that there are only finitely many injections $H_{1} \hookrightarrow H_{2}$ whose image has index $\leq N$, up to postcomposition with an inner automorphism of $H_{2}$. This is clear since $H_{2}$ has finitely many subgroups of index $\leq N$ and $\operatorname{Out}\left(H_{1}\right)$ is finite.

Since $P \mathscr{D}$ is a locally finite complex (see Section 5 ) and every closed simplex contains a reduced tree, it suffices to show that there are finitely many $\operatorname{Out}_{\mathscr{D}}(G)$-orbits of non-metric reduced trees. As $\mathscr{D}$ is non-ascending, all reduced trees in $\mathcal{D}$ are related by slide moves (Theorem 7.2), so there are finitely many possibilities for $\Gamma=T / G$ as a graph. We now have to consider $\Gamma$ as a graph of groups, with edge groups, vertex groups, and inclusions.

All reduced trees have the same edge and vertex stabilizers (Corollary 7.3), so there are finitely many possibilities for the isomorphism type of edge and vertex groups of $\Gamma$. As for the inclusions, we have observed that there are finitely many possibilities, up to inner automorphisms of vertex groups. But composing an inclusion with such an automorphism does not change the Bass-Serre tree [1], so we have shown that $\mathscr{D}$ only meets finitely many $\operatorname{Out}(G)$-orbits of non-metric reduced trees. Assertion (3) follows.

All finiteness conditions (but not smallness) are satisfied when $G$ is a "generic" hyperbolic group (one whose boundary is a Menger curve). When all conditions are satisfied, $\operatorname{Out}(G)$ acts on the contractible complex $P \mathscr{D}$ with finite stabilizers and finitely many orbits of simplices, so is $F_{\infty}$. We have for instance: 
Corollary 8.7. Let $p$ be prime. Suppose that $T$ is an irreducible locally finite $G$ tree with stabilizers commensurable to $\mathrm{BS}(1, p)$. If there is no non-trivial integer modulus, then $\operatorname{Out}(G)$ is $F_{\infty}$.

Proof. $\mathrm{BS}(1, p)$ is small. Its subgroups of finite index are isomorphic to some $\mathrm{BS}\left(1, p^{n}\right)$, so have trivial center. Since $p$ is prime, their Out is finite by [10]. A group containing $\mathrm{BS}\left(1, p^{n}\right)$ with finite index also has Out finite (see e.g. [20], Lemma 5.4).

\section{References}

[1] H. Bass, Covering theory for graphs of groups. J. Pure Appl. Algebra 89 (1993), 3-47. Zbl 0805.57001 MR 1239551

[2] H. Bass and R. Kulkarni, Uniform tree lattices. J. Amer. Math. Soc. 3 (1990), 843-902. Zbl 0734.05052 MR 1065928

[3] M. Bestvina and M. Feighn, Bounding the complexity of simplicial group actions on trees. Invent. Math. 103 (1991), 449-469. Zbl 0724.20019 MR 1091614

[4] B. H. Bowditch, Cut points and canonical splittings of hyperbolic groups. Acta Math. 180 (1998), 145-186. Zbl 0911.57001 MR 1638764

[5] M. Bridson and A. Miller, Lost manuscript.

[6] I. Chiswell, Introduction to $\Lambda$-trees. World Scientific, Singapore 2001. Zbl 1004.20014 MR 1851337

[7] M. Clay, Contractibility of deformation spaces of G-trees. Algebr. Geom. Topol. 5 (2005), 1481-1503. Zbl 02221903 MR 2186106

[8] M. Clay, A fixed point theorem for deformation spaces of $G$-trees. Comment. Math. Helv. 82 (2007), 237-246.

[9] M. Clay, Deformation spaces of $G$-trees. PhD thesis, University of Utah, Salt Lake City 2006.

[10] D. J. Collins, The automorphism towers of some one-relator groups. Proc. London Math. Soc. (3) 36 (1978), 480-493. Zbl 0376.20025 MR 0470091

[11] M. Culler and J. W. Morgan, Group actions on $\mathbb{R}$-trees. Proc. London Math. Soc. (3) 55 (1987), 571-604. Zbl 0658.20021 MR 907233

[12] M. Culler and K. Vogtmann, Moduli of graphs and automorphisms of free groups. Invent. Math. 84 (1986), 91-119. Zbl 0589.20022 MR 830040

[13] M. Culler and K. Vogtmann, The boundary of outer space in rank two. In Arboreal group theory (Berkeley, CA, 1988), Math. Sci. Res. Inst. Publ. 19, Springer, New York 1991, 189-230. Zbl 0786.57002 MR 1105335

[14] W. Dicks, Groups, trees and projective modules. Lecture Notes in Math. 790, SpringerVerlag, Berlin 1980. Zbl 0427.20016 MR 0584790 
[15] M. J. Dunwoody and M. E. Sageev, JSJ-splittings for finitely presented groups over slender groups. Invent. Math. 135 (1999), 25-44. Zbl 0939.20047 MR 1664694

[16] M. Forester, Deformation and rigidity of simplicial group actions on trees. Geom. Topol. 6 (2002), 219-267. Zbl 02062447 MR 1914569

[17] M. Forester, On uniqueness of JSJ decompositions of finitely generated groups. Comment. Math. Helv. 78 (2003), 740-751. Zbl 1040.20032 MR 2016693

[18] M. Forester, Splittings of generalized Baumslag-Solitar groups. Geom. Dedicata 121 (2006), 43-59. Zbl 05119716

[19] K. Fujiwara and P. Papasoglu, JSJ-decompositions of finitely presented groups and complexes of groups. Geom. Funct. Anal. 16 (2006), 70-125. Zbl 1097.20037 MR 2221253

[20] V. Guirardel, G. Levitt, The outer space of a free product. Proc. London Math. Soc., to appear. arXiv:math.GR/0501288

[21] V. Guirardel and G. Levitt, A general construction of JSJ decompositions. In Proceedings of the Barcelona Conference on Geometric Group Theory 2005, to appear.

http://picard.ups-tlse.fr/ guirardel/papiers/Barcelone.pdf

[22] V. Guirardel and G. Levitt, in preparation.

[23] S. Krstić and K. Vogtmann, Equivariant outer space and automorphisms of free-by-finite groups. Comment. Math. Helv. 68 (1993), 216-262. Zbl 0805.20030 MR 1214230

[24] G. Levitt, Automorphisms of hyperbolic groups and graphs of groups. Geom. Dedicata 114 (2005), 49-70. Zbl 02227554 MR 2174093

[25] G. Levitt, On the automorphism group of generalized Baumslag-Solitar groups. Geom. Topol., to appear; arXiv:math.GR/0511083

[26] D. McCullough and A. Miller, Symmetric automorphisms of free products. Mem. Amer. Math. Soc. 122 (1996). Zbl 0860.20029 MR 1329943

[27] J. W. Morgan and P. B. Shalen, Valuations, trees, and degenerations of hyperbolic structures, I. Ann. of Math. (2) 120 (1984), 401-476. Zbl 0583.57005 MR 769158

[28] F. Paulin, The Gromov topology on $\mathbb{R}$-trees. Topology Appl. 32 (1989), 197-221. Zbl 0675.20033 MR 1007101

[29] F. Paulin, Sur les automorphismes extérieurs des groupes hyperboliques. Ann. Sci. École Norm. Sup. (4) 30 (1997), 147-167. Zbl 0877.20014 MR 1432052

[30] E. Rips and Z. Sela, Cyclic splittings of finitely presented groups and the canonical JSJ decomposition. Ann. of Math. (2) 146 (1997), 53-109. Zbl 0910.57002 MR 1469317

[31] P. Scott and G. A. Swarup, Regular neighbourhoods and canonical decompositions for groups. Astérisque 289 (2003). Zbl 1036.20028 MR 2032389

[32] Z. Sela, Acylindrical accessibility for groups. Invent. Math. 129 (1997), 527-565. Zbl 0887.20017 MR 1465334

[33] J.-P. Serre, Arbres, amalgames, SL2. Astérisque 46 (1977). Zbl 0369.20013 MR 0476875

[34] P. B. Shalen, Dendrology of groups: an introduction. In Essays in group theory, Math. Sci. Res. Inst. Publ. 8, Springer-Verlag, New York 1987, 265-319. Zbl 0649.20033 MR 19830

[35] R. Skora, Deformations of length functions in groups. Preprint 1989. 
[36] T. White, Fixed points of finite groups of free group automorphisms. Proc. Amer. Math. Soc. 118 (1993), 681-688. Zbl 0798.20021 MR 1164152

Received July 1, 2006; revised October 2, 2006

Laboratoire Émile Picard, UMR CNRS 5580, Université Paul Sabatier, 31062 Toulouse Cedex 4, France

E-mail: guirardel@math.ups-tlse.fr

Laboratoire de Mathématiques Nicolas Oresme, UMR CNRS 6139, BP 5186, Université de Caen, 14032 Caen Cedex, France

E-mail: levitt@math.unicaen.fr 